\title{
QUASI-ACTIONS ON TREES AND PROPERTY (QFA)
}

\author{
J. F. MANNING
}

with an appendix by N. Monod and B. Rémy

\begin{abstract}
We prove some general results about quasi-actions on trees and define Property (QFA), which is analogous to Serre's Property (FA), but in the coarse setting. This property is shown to hold for a class of groups, including $\operatorname{SL}(n, \mathbb{Z})$ for $n \geqslant 3$. We also give a way of thinking about Property (QFA) by breaking it down into statements about particular classes of trees.
\end{abstract}

\section{Introduction}

Group quasi-actions are a natural coarse generalization of isometric group actions (see Section 2 for precise definitions). The main motivating question for this paper is the following.

Question 1.1. What kind of finitely generated groups admit (or do not admit) nontrivial quasi-actions on trees?

Cobounded quasi-actions on bounded valence bushy trees were studied in [18], where it was shown that such quasi-actions are always quasi-conjugate to isometric actions on trees. The same is not true for quasi-actions on $\mathbb{R}$ or on infinite valence bushy trees. Part of the reason for this is that isometric actions on $\mathbb{R}$-trees are always quasi-conjugate to actions on simplicial trees, but this is not the complete story. Examples of quasi-actions on simplicial trees which are not quasi-conjugate to actions on $\mathbb{R}$-trees are given in [16]. Such 'exotic' quasi-actions on trees appear to be plentiful, but it is not clear how much information can be obtained from them. We hope to clarify the situation by offering some partial answers to Question 1.1.

Recall that a group $G$ is said to have Property (FA) if, for any isometric action of $G$ on a simplicial tree $T$, there is some fixed point for the action (that is, there is some point $x \in T$ so that the orbit $G x=\{x\})$.

Definition 1.2. We will say that a group $G$ has Property (QFA) if, for every quasi-action of $G$ on any tree $T$, there is some $x \in T$ so that the orbit $G x$ has finite diameter (equivalently, every orbit has finite diameter).

Here is a brief outline. Section 2 consists mainly of definitions and can probably be skipped by the expert. In Section 3 we prove some useful facts about quasi-actions on trees. In Section 4 we use these facts to prove Property (QFA) for a class of boundedly generated groups including $\operatorname{SL}(n, \mathcal{O})$, for $n \geqslant 3$ and $\mathcal{O}$ the ring of integers

Received 27 February 2004; revised 11 August 2004.

2000 Mathematics Subject Classification 20F65 (primary), 20E08, 53C23, 22E40 (secondary). 
of an algebraic number field. In Section 5 we try to understand Property (QFA) by breaking it into statements about different kinds of trees. An appendix by Nicolas Monod and Bertrand Rémy gives some examples of boundedly generated lattices in Lie groups which satisfy Kazhdan's Property (T) but not Property (QFA).

Unless otherwise stated, all groups are assumed to be finitely generated.

\section{Preliminaries}

\subsection{Coarse geometry}

Definition 2.1. If $X$ and $Y$ are metric spaces, $K \geqslant 1$ and $C \geqslant 0$, a $(K, C)$ quasi-isometric embedding of $X$ into $Y$ is a function $q: X \rightarrow Y$ so that for all $x_{1}, x_{2} \in X$

$$
\frac{1}{K} d\left(x_{1}, x_{2}\right)-C \leqslant d\left(q\left(x_{1}\right), q\left(x_{2}\right)\right) \leqslant K d\left(x_{1}, x_{2}\right)+C .
$$

If in addition the map $q$ is $C$-coarsely onto (that is, every $y \in Y$ is distance at most $C$ from some point in $q(X))$, then $q$ is called a $(K, C)$-quasi-isometry. The two metric spaces $X$ and $Y$ are then said to be quasi-isometric to one another. This is a symmetric condition.

Definition 2.2. A $(K, C)$-quasi-geodesic in $X$ is a $(K, C)$-quasi-isometric embedding $\gamma: \mathbb{R} \rightarrow X$. We will occasionally abuse notation by referring to the image of $\gamma$ as a quasi-geodesic.

Definition 2.3. A $(K, C)$-quasi-action of a group $G$ on a metric space $X$ is a map $A: G \times X \rightarrow X$, denoted $A(g, x) \mapsto g x$, so that the following hold:

(i) for each $g, A(g,-): G \rightarrow G$ is a $(K, C)$-quasi-isometry;

(ii) for each $x \in X$ and $g, h \in G$, we have

$$
d(g(h x),(g h) x)=d(A(g, A(h, x)), A(g h, x)) \leqslant C .
$$

(Note that $K$ and $C$ must be independent of $g$ and $h$.) We call a quasi-action cobounded if, for every $x \in X$, the map $A(-, x): G \rightarrow X$ is $C^{\prime}$-coarsely onto for some $C^{\prime}$.

Definition 2.4. Suppose that $A_{X}: G \times X \rightarrow X$ and $A_{Y}: G \times Y \rightarrow Y$ are quasi-actions. A map $f: X \rightarrow Y$ is called coarsely equivariant if there is some $C$ so that $d\left(f \circ A_{X}(g, x), A_{Y}(g, f(x))\right) \leqslant C$ for all $g$ in $G$ and $x$ in $X$.

A coarsely equivariant quasi-isometry is called a quasi-conjugacy.

EXAMPLE 2.5. Let $f: G \rightarrow \mathbb{R}$ be a quasicharacter; that is, suppose that for some $C \geqslant 0$ and for all $g$ and $h$ in $G,|\delta f(g, h)|=|f(g h)-f(g)-f(h)| \leqslant C$ (see also Section 2.3). A $(1, C)$-quasi-action of $G$ on $\mathbb{R}$ is given by $A(g, x)=x+f(g)$.

\subsection{Quasi-trees and other hyperbolic spaces}

All metric spaces will be assumed to be complete geodesic metric spaces, and the distance between two points $x$ and $y$ will usually be denoted $d(x, y)$. Several equivalent definitions and a much fuller discussion of $\delta$-hyperbolic metric spaces can 
be found, for instance, in [4, Chapter III.H]. We will use a definition which emphasizes the 'arboreality' of hyperbolic spaces. Given a geodesic triangle $\Delta(x, y, z)$ in any metric space, there is a unique comparison tripod, $T_{\Delta}$, a metric tree so that the distances between the three extremal points of the tree, $\bar{x}, \bar{y}$ and $\bar{z}$, are the same as the distances between $x, y$ and $z$ (see Figure 1). There is an obvious map $\pi: \Delta(x, y, z) \rightarrow T_{\Delta}$ which takes $x$ to $\bar{x}, y$ to $\bar{y}$ and $z$ to $\bar{z}$, and which is an isometry on each side of $\Delta(x, y, z)$.
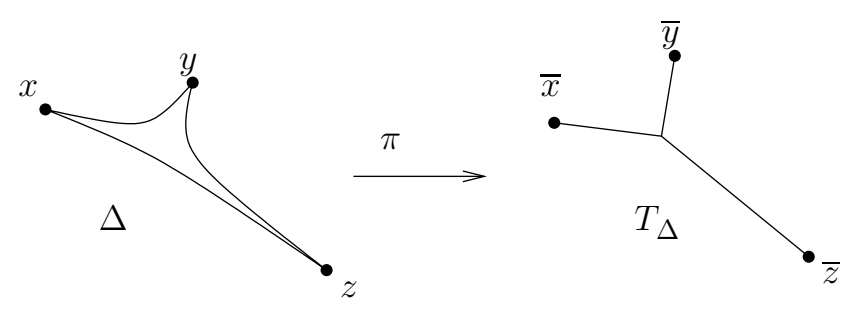

Figure 1. A triangle and its comparison tripod.

Definition 2.6. A space $X$ is $\delta$-hyperbolic if for any geodesic triangle $\Delta(x, y, z)$ and any point $p$ in the comparison tripod $T_{\Delta}$, the diameter of $\pi^{-1}(p)$ is less than $\delta$. If $\delta$ is unimportant we may simply say that $X$ is Gromov hyperbolic.

Definition 2.7. Let $x, y, z \in X$. The Gromov product of $x$ and $y$ with respect to $z$ is $(x, y)_{z}=\frac{1}{2}(d(x, z)+d(y, z)-d(x, y))$. Equivalently, $(x, y)_{z}$ is the distance from $\bar{z}$ to the central vertex of the comparison tripod $T_{\Delta}$ for any geodesic triangle $\Delta(x, y, z)$.

The following is well known (see for example [4, III.H.1.22]).

LEMma 2.8. For any $\delta$ there is some $\delta^{\prime}$ so that if $x, y, z$, and $w$ are points in a $\delta$-hyperbolic space, then

$$
(x, y)_{w} \geqslant \min \left\{(x, z)_{w},(y, z)_{w}\right\}-\delta^{\prime} .
$$

Definition 2.9. Fix some $z \in X$. We say that a sequence $\left\{x_{i}\right\}$ tends to infinity if $\liminf \operatorname{in}_{i, j \rightarrow \infty}\left(x_{i}, x_{j}\right)_{z}=\infty$. On the set of such sequences we may define an equivalence relation: $\left\{x_{i}\right\} \sim\left\{y_{i}\right\}$ if $\liminf _{i, j \rightarrow \infty}\left(x_{i}, y_{i}\right)_{z}=\infty$. The Gromov boundary of $X$, also written $\partial X$, is the set of equivalence classes of sequences tending to infinity. The Gromov boundary does not depend on the choice of $z$.

REMARK 2.10. We may topologize $X \cup \partial X$ so that if $\left\{x_{i}\right\}$ tends to infinity then $\lim _{i \rightarrow \infty} x_{i}=\left[\left\{x_{i}\right\}\right]$. Furthermore, if $\gamma:[0, \infty) \rightarrow X$ is a quasi-geodesic ray, then for any sequence $\left\{t_{i}\right\}$ with $\lim _{i \rightarrow \infty} t_{i}=\infty$, the sequence $\left\{\gamma\left(t_{i}\right)\right\}$ tends to infinity. The point $\left\{\gamma\left(t_{i}\right)\right\} \in \partial X$ does not depend on the choice of $\left\{t_{i}\right\}$. 
The following lemma about 'stability' of quasi-geodesics is well known. We include a proof for completeness and because we were unable to find this precise statement in the literature (but see Remark 2.12).

Lemma 2.11. Let $K \geqslant 1, C \geqslant 0, \delta \geqslant 0$. Then there is some $B=B(K, C, \delta)$ so that if $\gamma$ and $\gamma^{\prime}$ are two $(K, C)$-quasi-geodesics with the same endpoints in $X \cup \partial X$, and $X$ is a $\delta$-hyperbolic geodesic metric space, then the image of $\gamma$ lies in a $B$-neighborhood of the image of $\gamma^{\prime}$.

Proof. Fix $K, C$, and $\delta$. Several proofs of the lemma exist in the literature under the assumption that $\gamma$ and $\gamma^{\prime}$ are quasi-geodesics of finite length ([4, III.H.1.7] for example). Let $B_{0}=B_{0}(K, C, \delta)$ be the constant which suffices in this case.

Suppose first that $\gamma:[0, \infty) \rightarrow X$ and $\gamma^{\prime}:[0, \infty) \rightarrow X$ are quasi-geodesic rays, sharing one endpoint in $X$ and another in $\partial X$. Let $z=\gamma(0)=\gamma^{\prime}(0)$. For each integer $i>0$ let $x_{i}$ be the first point in the image of $\gamma$ which is a distance $i$ from $z$, and let $y_{i}$ be the first point in the image of $\gamma^{\prime}$ which is a distance $i$ from $z$. We have $\left\{x_{i}\right\} \sim\left\{y_{i}\right\}$ and so in particular $\left(x_{i}, y_{i}\right)_{z} \rightarrow \infty$ as $i \rightarrow \infty$.

Note that there is an $\epsilon$ depending only on $K, C$, and $\delta$ so that the image of $\gamma$ is contained in an $\epsilon$-neighborhood of $\left\{x_{i} \mid i \in \mathbb{N}\right\}$ and the image of $\gamma^{\prime}$ is contained in an $\epsilon$-neighborhood of $\left\{y_{i} \mid i \in \mathbb{N}\right\}$. (It is sufficient to bound $d\left(x_{i}, x_{i+1}\right)$. Let $p$ be a point on $\left[z, x_{i+1}\right]$ so that $d\left(x_{i}, p\right) \leqslant B_{0}$. Then

$$
d\left(x_{i}, x_{i+1}\right) \leqslant d\left(p, x_{i}\right)+d\left(p, x_{i+1}\right) \leqslant B_{0}+d\left(p, x_{i+1}\right) .
$$

However, since $d(p, z)+d\left(p, x_{i}\right) \geqslant d\left(x_{i}, z\right)=i$ we have

$$
d\left(p, x_{i+1}\right)=i+1-d(p, z) \leqslant i+1-\left(i-B_{0}\right)=B_{0}+1,
$$

which implies that $d\left(x_{i}, x_{i+1}\right) \leqslant 2 B_{0}+1$. $)$

Thus to prove the lemma, it suffices to bound $d\left(x_{i}, y_{i}\right)$ in terms of $K, C$, and $\delta$. Fixing $i$, choose $N$ so that $\left(x_{N}, y_{N}\right)_{z}>i+B_{0}+2 \delta$ (see Figure 2). Let $p_{x}$ be some point on $\left[z, x_{N}\right]$ with $d\left(p_{x}, x_{i}\right) \leqslant B_{0}$, and let $p_{y}$ be some point on $\left[z, y_{N}\right]$ with $d\left(p_{y}, y_{i}\right) \leqslant B_{0}$. Note that $d\left(z, p_{x}\right)$ and $d\left(z, p_{y}\right)$ are both at most $i+B_{0}$, and hence less than $\left(x_{N}, y_{N}\right)_{z}$. Consider the comparison tripod $T$ for a geodesic triangle with vertices $\left\{z, x_{N}, y_{N}\right\}$. Both $\overline{p_{x}}$ and $\overline{p_{y}}$ lie in the leg of the tripod nearest $\bar{z}$, as the length of this leg is precisely $\left(x_{N}, y_{N}\right)_{z}$. (If $\xi$ is a point in the geodesic triangle, we write $\bar{\xi}$ for the corresponding point in $T$.) Thus

$$
d\left(\overline{p_{x}}, \overline{p_{y}}\right)=\left|d\left(p_{x}, z\right)-d\left(p_{y}, z\right)\right| \leqslant 2 B_{0} .
$$

However, this implies that $d\left(p_{x}, p_{y}\right) \leqslant 2 B_{0}+\delta$, from which it follows that $d\left(x_{i}, y_{i}\right) \leqslant$ $4 B_{0}+\delta$. It follows that any point on $\gamma$ is within $B_{1}(K, C, \delta)=4 B_{0}+\delta+\epsilon$ of the $\gamma^{\prime}$.

Finally, suppose that both endpoints of $\gamma$ and $\gamma^{\prime}$ are in $\partial X$ and that $\lim _{t \rightarrow \infty}$ $\gamma(t)=\lim _{t \rightarrow \infty} \gamma^{\prime}(t)$ and $\lim _{t \rightarrow-\infty} \gamma(t)=\lim _{t \rightarrow-\infty} \gamma^{\prime}(t)$. Let $D=\inf _{s, t} d(\gamma(s)$, $\left.\gamma^{\prime}(t)\right)$ and re-parameterize $\gamma$ and $\gamma^{\prime}$ so that $d\left(\gamma(0), \gamma^{\prime}(0)\right) \leqslant D+1$. It is not hard to see that $\gamma$ must lie in a $B_{1}(K, C+D+1, \delta)$-neighborhood of $\gamma^{\prime}$. Thus if we can find a universal bound $D_{\max }$ for $D$, we may set $B(K, C, \delta)=B_{1}\left(K, C+D_{\max }+1, \delta\right)$ and the lemma will be proved.

Since $\liminf \operatorname{in}_{s, t \rightarrow \infty}\left(\gamma(s), \gamma^{\prime}(t)\right)_{\gamma(0)}=\infty$ we may choose $s$ and $t$ so that

$$
\left(\gamma(s), \gamma^{\prime}(t)\right)_{\gamma(0)} \geqslant D+1 \text {. }
$$




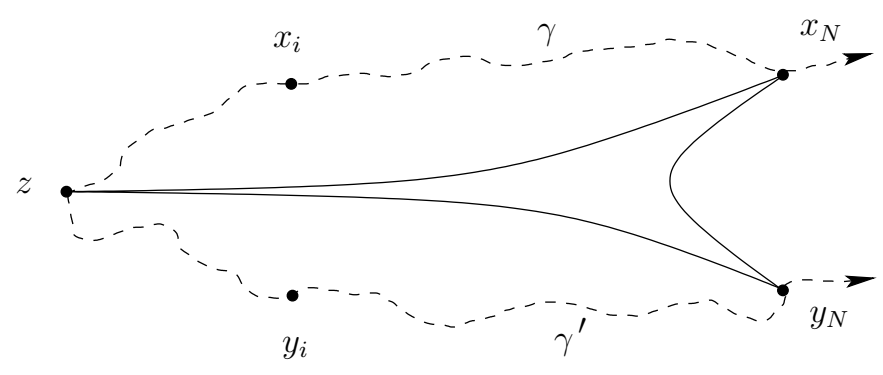

Figure 2. Closeness of quasi-geodesic rays follows from closeness of long quasi-geodesic segments.

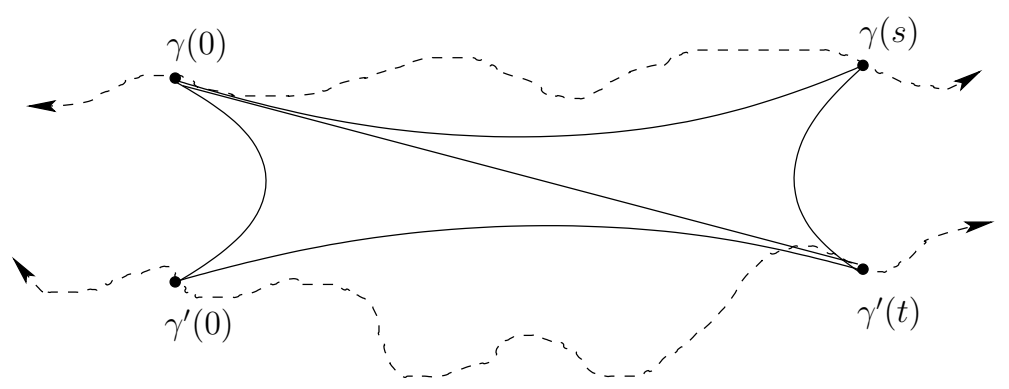

FiguRE 3. If $\left(\gamma(s), \gamma^{\prime}(t)\right)_{\gamma(0)}$ is large enough, the geodesics must come close together.

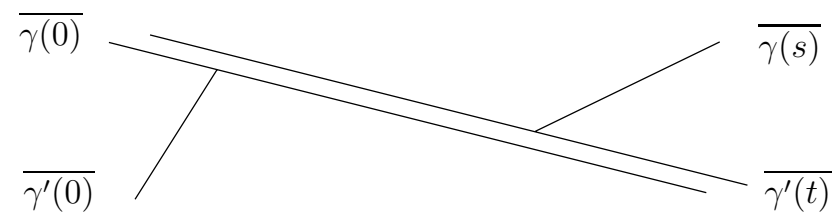

Figure 4. The comparison tripods for the geodesic triangles in Figure 3 must fit together as shown, since $\left(\gamma(s), \gamma^{\prime}(0)\right)_{\gamma(0)} \leqslant D+1$, but $\left(\gamma(s), \gamma^{\prime}(t)\right)_{\gamma(0)} \geqslant D+1$.

In this case any geodesic arcs between $\gamma(0)$ and $\gamma(s)$ and between $\gamma^{\prime}(0)$ and $\gamma^{\prime}(t)$ must contain points which are at most $2 \delta$ apart (see Figure 3 and the comparison tripods in Figure 4). There must therefore be points on the quasi-geodesic segments $\left.\gamma\right|_{[0, s]}$ and $\left.\gamma^{\prime}\right|_{[0, t]}$ which are within $2 \delta+B_{0}(K, C, \delta)$ of one another, and so $D_{\max } \leqslant$ $2 \delta+B_{0}(K, C, \delta)$.

REMARK 2.12. A Gromov hyperbolic space $X$ is called ultra-complete if every two points in $X \cup \partial X$ are joined by a geodesic. Trees are always ultra-complete, but general locally infinite Gromov hyperbolic graphs need not be. It is claimed in [11, Section 7.5] that any $\delta$-hyperbolic geodesic metric space $X$ isometrically embeds in an ultra-complete space $Y$ with $\sup _{y \in Y} d(y, X) \leqslant C$ for some $C<\infty$. Another way 
to prove Lemma 2.11 would be to show first that $C$ depends only on $\delta$ and then apply [7, Théorème 3.1].

Definition 2.13. Fix $x \in X$, where $X$ is a $\delta$-hyperbolic metric space on which $G$ quasi-acts. Let $O_{g, x}: \mathbb{R} \rightarrow X$ be defined by $O_{g, x}(t)=g^{\lfloor t\rfloor} x$, where $\lfloor t\rfloor$ is the largest integer smaller than $t$. If $O_{g, x}$ has bounded image, we say $g$ quasi-acts elliptically. If $O_{g, x}$ is a quasi-geodesic, then we say $g$ quasi-acts hyperbolically. (If $G$ acts isometrically on $X$, we may simply say $g$ acts elliptically or hyperbolically.)

It is not hard to check that Definition 2.13 is independent of $x$ and agrees with the standard definitions in case $G$ acts isometrically. It has the added benefit of being invariant under quasi-conjugacy. In the case that $G$ acts isometrically on $X$, and $g \in G$ acts hyperbolically, then $g$ always has a quasi-axis, a quasi-geodesic whose image is invariant under the infinite cyclic group $\langle g\rangle$. Indeed if $x \in X$, and $\gamma_{0}:[0,1] \rightarrow X$ is a geodesic segment with $\gamma_{0}(0)=x$ and $\gamma_{0}(1)=g x$, then the reader can easily verify that $\gamma: \mathbb{R} \rightarrow X$ is a continuous quasi-geodesic, if we define $\gamma(t)=g^{\lfloor t\rfloor} \gamma_{0}(t-\lfloor t\rfloor)$.

EXAMPLE 2.14. If the quasicharacter $f: G \rightarrow \mathbb{R}$ from Example 2.5 is a homomorphism restricted to each cyclic subgroup, it is called a pseudocharacter or homogeneous quasicharacter. As in Example 2.5, the pseudocharacter $f$ induces a quasi-action of $G$ on $\mathbb{R}$. A group element $g \in G$ quasi-acts hyperbolically if and only if $f(g)$ is nonzero. Unless $f$ is identically 0 , the quasi-action is cobounded.

Definition 2.15. A quasi-tree is a complete geodesic metric space quasiisometric to some simplicial tree. (All simplicial trees are assumed to be endowed with a path metric in which every edge has length 1.)

Quasi-trees satisfy a particularly strong form of $\delta$-hyperbolicity.

Lemma 2.16. If $X$ is a quasi-tree, then there is a $\delta>0$ so that:

(i) for any two points $x$ and $y$ in $X$, and any point $p$ on a geodesic between $x$ and $y$, any path from $x$ to $y$ must pass within $\delta$ of $p$;

(ii) $X$ is $\delta$-hyperbolic.

Proof. This is left as an exercise for the reader.

Definition 2.17. If a quasi-tree $X$ satisfies the conclusions of Lemma 2.16 for $\delta \geqslant 0$, we say that $X$ is a $\delta$-quasi-tree.

\subsection{Bounded cohomology, amenability, and Trauber's Theorem}

We give only a few needed facts here. For fuller discussion of these topics, see $[\mathbf{9}]$ and $[\mathbf{1 0}]$.

We give the definition of bounded cohomology for groups only. 
Definition 2.18. The bounded cohomology $H_{b}^{*}(G ; \mathbb{R})$ of a group $G$ is the cohomology of the cochain complex $C_{b}^{*}(G ; \mathbb{R})$, where

$$
C_{b}^{n}(G ; \mathbb{R})=\left\{f: G^{n} \rightarrow \mathbb{R}\left|\sup _{G^{n}}\right| f\left(g_{1}, \ldots, g_{n}\right) \mid<\infty\right\}
$$

and $\delta: C_{b}^{n}(G ; \mathbb{R}) \rightarrow C_{b}^{n+1}(G ; \mathbb{R})$ is given by

$$
\begin{aligned}
\delta f\left(g_{1}, \ldots, g_{n+1}\right)= & f\left(g_{2}, \ldots, g_{n+1}\right)+\sum_{i=1}^{n}(-1)^{i} f\left(g_{1}, \ldots, g_{i-1}, g_{i} g_{i+1}, \ldots, g_{n+1}\right) \\
& +(-1)^{n+1} f\left(g_{1}, \ldots, g_{n}\right) .
\end{aligned}
$$

This cochain complex is a subcomplex of the complex $C^{*}(G ; \mathbb{R})$ of all real valued functions on $G, G \times G$, and so on. The cohomology of $C^{*}(G ; \mathbb{R})$ is the ordinary cohomology of $G$ with real coefficients.

Definition 2.19. A quasicharacter is an element $f$ of $C^{1}(G ; \mathbb{R})$ whose coboundary $\delta f$ lies in $C_{b}^{2}(G ; \mathbb{R})$. The quasicharacter $f$ is a pseudocharacter if in addition $f\left(g^{n}\right)=n f(g)$ for all $n \in \mathbb{Z}$ and $g \in G$. In either case we define the defect of $f$ as $\|\delta f\|=\sup _{g, h \in G}|\delta f(g, h)|$.

REMARK 2.20. Note that if $f$ is a quasicharacter, and $\phi$ is given by $\phi(g)=$ $\lim _{n \rightarrow \infty} f\left(g^{n}\right) / n$, then $\phi$ is a pseudocharacter with $\phi-f$ bounded and $[\delta \phi]=[\delta f]$. If a pseudocharacter $\phi$ is ever nonzero, then it is unbounded.

The relationship between quasicharacters and pseudocharacters and bounded cohomology is a major tool for understanding $H_{b}^{2}$ in certain situations (see for example $[\mathbf{2}]$ and $[\mathbf{9}])$.

We will need only a few facts about amenable groups. First, nilpotent groups are amenable. Second, amenable groups contain no free subgroups. Third, we have the following theorem.

TheOREM 2.21 (Trauber's Theorem). If $G$ is an amenable group then $H_{b}^{n}(G ; \mathbb{R})=0$ for all $n$.

For a definition of amenability and a proof of Theorem 2.21 see [9].

\section{Lemmata}

This section contains some general results about quasi-actions on trees by (finitely generated) groups. The key idea is that quasi-actions on trees and isometric actions on quasi-trees are essentially equivalent. Proposition 3.1 gives a way to replace a quasi-action on a tree by an isometric action on a Cayley graph which is a quasitree. In Section 3.2 it is shown that there is no such thing as a 'parabolic' isometry of a quasi-tree. In Section 3.3 we show how to obtain a pseudocharacter from a quasi-action on a tree which fixes one end.

\subsection{Getting some action}

Recall that if $G$ is a group and $S$ some (not necessarily finite) generating set, then we may form the Cayley graph $\Gamma(G, S)$ by setting the zero-skeleton $\Gamma(G, S)^{0}=G$ 
and connecting $g$ to $g s$ with an edge whenever $s \in S$. We make $\Gamma(G, S)$ a metric space with a path metric in which every edge has length 1 . Then $G$ acts on the left by isometries of $\Gamma(G, S)$.

Proposition 3.1. Suppose a finitely generated group $G$ quasi-acts on a simplicial tree $T$. Then there is a generating set $S$ for $G$ so that the Cayley graph $\Gamma(G, S)$ embeds coarsely equivariantly and quasi-isometrically in $T$. Specifically, for $x \in T$ and $R$ sufficiently large, we may take $S=\{s \in G \mid d(s(x), x) \leqslant R\}$.

Proof. We suppose that $G$ has a $(K, C)$-quasi-action on the simplicial tree $T$. Let $S_{0}$ be a finite generating set for $G$, and let $\Gamma_{0}=\Gamma\left(G, S_{0}\right)$ be the associated Cayley graph. Fix $x \in T$ and define $\pi_{0}: \Gamma_{0} \rightarrow T$ by $\pi_{0}(g)=g x$. We may assume $\pi_{0}$ sends each edge to a geodesic. For $s \in S_{0}$ and $g \in G$ we have

$$
d(g x,(g s) x) \leqslant d(g x, g(s x))+C \leqslant K d(x, s x)+2 C .
$$

Let $D=K\left(\sup _{s \in S_{0}} d(x, s x)\right)+2 C$. We fix $R \geqslant 2 K D+K C$.

We now set $S=\{s \in G \mid d(s(x), x) \leqslant R\}$, as in the statement of the proposition. We let $\Gamma=\Gamma(G, S)$ and extend $\pi_{0}$ to $\pi: \Gamma \rightarrow T$ which we may also assume sends each edge to a geodesic. To show that $\pi$ is a quasi-isometric embedding, we must bound $d(\pi(p), \pi(q))$ above and below by affine functions of $d(p, q)$. We may restrict our attention to the case when both $p$ and $q$ are vertices (group elements), as $\Gamma$ is quasi-isometric to its zero-skeleton.

Suppose that $d(p, q)=1$. Then there is some $s \in S$ so that $p=q s$ and so

$$
d(p x, q x)=d(q s x, q x) \leqslant d(q(s x), q x)+C \leqslant K d(s x, x)+2 C \leqslant K R+2 C .
$$

In general we have $d(\pi(p), \pi(q))=d(p x, q x) \leqslant(K R+2 C) d(p, q)$.

In the other direction, note first that if $d(\pi(p), \pi(q))<R / K-C$, then $d(p, q)=1$. If on the other hand $d(\pi(p), \pi(q)) \geqslant R / K-C$, we will choose a new $p^{\prime}$ so that $d\left(p, p^{\prime}\right)=1$ and $\pi\left(p^{\prime}\right)$ is closer to $\pi(q)$ than $\pi(p)$ was. Note that $d(\pi(p), \pi(q)) \geqslant$ $R / K-C$ implies that $d(\pi(p), \pi(q)) \geqslant 2 D$ by our choice of $R$. Let $z$ be the point on the geodesic $[\pi(p), \pi(q)]$ which is a distance of $3 D / 2$ from $\pi(p)$. This point is in the image of $\Gamma(G, S)$, and so there is a group element $p^{\prime}$ so that $d\left(\pi\left(p^{\prime}\right), z\right) \leqslant D / 2$. Since $d\left(p^{\prime} x, p x\right) \leqslant 2 D$ we have $d\left(p^{-1} p^{\prime} x, x\right) \leqslant 2 K D+C<R$, implying that $p^{-1} p^{\prime} \in S$ and so $d\left(p^{\prime}, p\right)=1$. On the other hand, $\pi\left(p^{\prime}\right)$ is at least $D$ closer to $\pi(q)$ than $\pi(p)$ is. Thus we can travel from $p$ to $q$ in $\Gamma$ by traversing at most $d(p x, q x) / D+1$ edges. In other words, $d(p, q) \leqslant d(p x, q x) / D+1$ or $d(\pi(p), \pi(q))=d(p x, q x) \geqslant \operatorname{Dd}(p, q)-D$.

Since

$$
\operatorname{Dd}(p, q)-D \leqslant d(\pi(p), \pi(q)) \leqslant(K R+2 C) d(p, q),
$$

the map $\pi$ is a quasi-isometric embedding.

We now show $\pi$ is coarsely equivariant. Again, we may restrict attention to vertices of $X$. Let $p$ be a vertex of $X$, and let $g \in G$. We need a universal bound on $d(g(\pi(p)), \pi(g(p)))$. Since $p$ is a vertex of $X$ it is a group element, and so $\pi(g(p))=\pi(g p)=(g p) x$ and $\pi(p)=p x$. By the definition of a $(K, C)$-quasiaction, $d(g(\pi(p)), \pi(g(p)))=d(g(p x),(g p) x) \leqslant C$, and so $\pi$ is coarsely equivariant.

Remark 3.2. Note that the isometric action we obtain from Proposition 3.1 is quasi-conjugate to the original quasi-action only in the cobounded case (compare 
Proposition 4.4 of [16]). This disadvantage is balanced by the fact that we may now work with a left-invariant metric on $G$ itself. Whether or not the original quasi-action is cobounded, the Cayley graph $\Gamma(G, S)$ is quasi-isometric to its image in $T$ and is hence a quasi-tree. Conversely, if $G$ acts by isometries on a quasi-tree, then there is a quasi-conjugate quasi-action on a tree.

\subsection{Cayley graphs of $\mathbb{Z}$}

The aim in this subsection is to explain why every element of a group quasiacting on a tree must quasi-act either hyperbolically or elliptically, in the sense of Definition 2.13. Suppose $g \in G$ and that $G$ quasi-acts on the tree $T$. Then the integers also quasi-act on $T$, via $n(x)=g^{n} x$ for $n \in \mathbb{Z}$. By Proposition 3.1, there is therefore a coarsely equivariant quasi-isometric embedding of some Cayley graph $\Gamma=\Gamma(\mathbb{Z}, S)$ into $T$. In particular this Cayley graph must be $\delta$-hyperbolic for some $\delta$. The element $g$ quasi-acts elliptically if and only if the diameter of $\Gamma$ is finite, and quasi-acts hyperbolically if and only if $S$ is finite. The following proposition shows that there are no other possibilities.

Proposition 3.3. Suppose that $\Gamma=\Gamma(\mathbb{Z}, S)$ is $\delta$-hyperbolic. Then either $S$ is finite or the diameter of $\Gamma$ is finite.

Proof. Let $\Delta \geqslant \max \left\{\delta, \delta^{\prime}, 1\right\}$, where $\delta^{\prime}$ is the constant from Lemma 2.8. We will write $|n|_{S}$ for the distance $d(0, n)$ in $\Gamma$. Assuming that the diameter if $\Gamma$ is infinite, we fix some $N>0$ so that $|N|_{S} \geqslant 10 \Delta$. For $k \in \mathbb{Z}$, let $D_{k}=|k N|_{S}$. Note that $D_{-k}=D_{k}$ for any $k$.

To complete the proof, we need the following claim.

\section{Claim 3.4. $D_{k} \geqslant|k|\left(|N|_{S}-4 \Delta\right)$.}

Proof. It suffices to prove the claim for all positive $k$. We inductively argue that the following two assertions hold for each $k$ :

$P_{k}: D_{k} \geqslant k\left(|N|_{S}-4 \Delta\right)$.

$Q_{k}:(-N,(k N))_{0} \leqslant 2 \Delta$.

The statement $P_{1}$ is obvious; the statement $Q_{1}$ can be proved as follows. Let $\sigma$ be a geodesic segment from $-N$ to 0 , and let $T$ be a geodesic triangle two of whose sides are $\sigma$ and $\sigma+N$. Let $z$ be a point on $\sigma$ so that $d(z, 0)=(-N, N)_{0}$ (see Figure 5). Then $d(z, z+N)$ is at most $|N|-2(-N, N)_{0}+\Delta$. On the other hand, since $N$ moves every vertex of $\Gamma(\mathbb{Z}, S)$ the same distance, we must have $d(z, z+N) \geqslant|N|-1$ ( $z$ may lie in the middle of an edge). Combining these two inequalities gives us $2(-N, N)_{0} \leqslant \Delta+1$, and so

$$
(-N, N)_{0} \leqslant \Delta \leqslant 2 \Delta
$$

(assertion $\left.Q_{1}\right)$.

Assuming $k \geqslant 2$, we argue the inductive step as follows. Note first that $D_{k}=$ $d(-N,(k-1) N)=|N|_{S}+|(k-1) N|_{S}-2(-N,(k-1) N)_{0}$. Since $(-N,(k-1) N) \leqslant 2 \Delta$ by the induction hypothesis $Q_{k-1}$, we have $D_{k} \geqslant D_{k-1}+|N|_{S}-4 \Delta$. Applying $P_{k-1}$ yields $D_{k} \geqslant k\left(|N|_{S}-4 \Delta\right)$, and the assertion $P_{k}$ is proved.

To prove the statement $Q_{k}$, consider four geodesic triangles as pictured in Figure 6 and the corresponding comparison tripods shown in Figure 7. The geodesic segment 


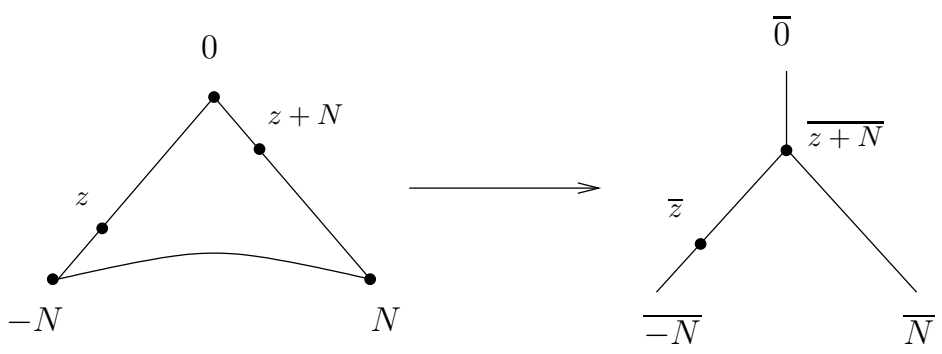

Figure 5. Bounding $(-N, N)_{0}$.

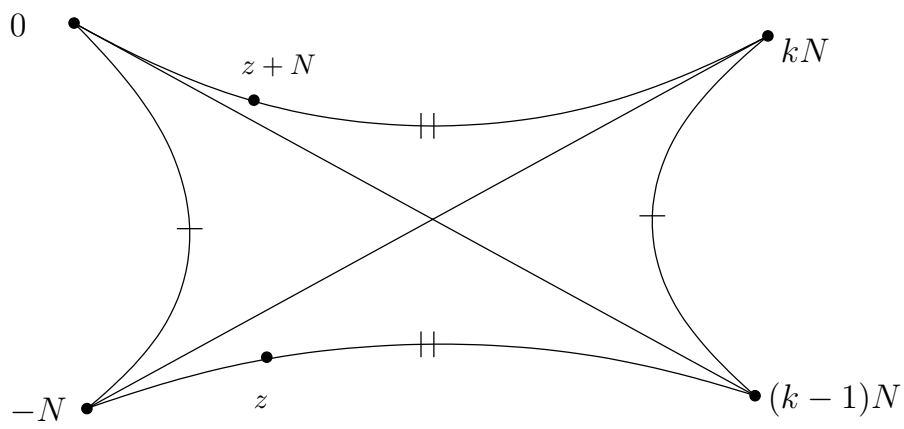

FiguRE 6. Induction step.
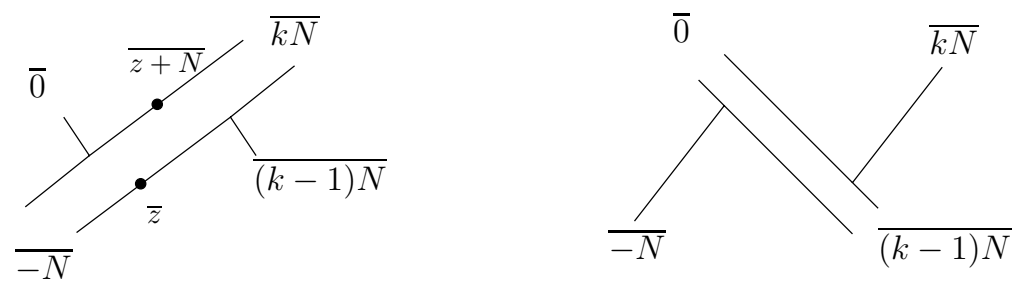

Figure 7. Comparison tripods for Figure 6. The tripods on the left are congruent to one another, as are those on the right.

between $-N$ and $(k-1) N$ in Figure 6 should be chosen to be the obvious translate of the one between 0 and $k N$. Lemma 2.8 implies that

$$
(-N,(k-1) N)_{0}+\Delta \geqslant \min \left\{(-N, k N)_{0},(k N,(k-1) N)_{0}\right\} .
$$

By assertion $Q_{k-1}$, the left hand side of equation (3.1) can be no larger than $3 \Delta$. It follows from the congruence of the two right hand tripods in Figure 7 that $(k N,(k-1) N)_{0}=D_{k-1}-(k N, 0)_{(k-1) N}=D_{k-1}-(-N,(k-1) N)_{0}$. Thus by $Q_{k-1}$ and $P_{k-1},(k N,(k-1) N)_{0}$ is at least $4 \Delta$ and so equation (3.1) implies that $(-N, k N)_{0} \leqslant 3 \Delta$. This does not yet establish $Q_{k}$, but it at least shows that the comparison tripods in the left half of Figure 7 are qualitatively correct; since $|N|_{S} \geqslant$ $2(-N, k N)_{0}$ and $k N>|N|_{S}$, the lengths of the three legs of the leftmost tripod 
must be ordered:

$$
(-N, k N)_{0} \leqslant(0, k N)_{-N} \leqslant(0,-N)_{k N}
$$

Now let $z$ be the point on $[-N,(k-1) N]$ so that $d(z,-N)=(0, k N)_{-N}$. Then we have

$$
|N|_{S}-1 \leqslant d(z, z+N) \leqslant 2 \Delta+(0, k N)_{-N}-(-N, k N)_{0}
$$

Since $(0, k N)_{-N}=|N|_{S}-(-N, k N)_{0}$, equation (3.2) may be rewritten as

$$
|N|_{S}-1 \leqslant 2 \Delta+|N|_{S}-2(-N, k N)_{0},
$$

which can be rearranged as $(-N, k N)_{0} \leqslant \Delta+\frac{1}{2} \leqslant 2 \Delta$, and $Q_{k}$ is proved. The proof of the claim in finished.

We now show that $S$ is finite. Suppose $s \in S$. Then $|s N|_{S} \leqslant N$. However, by the claim, $|s N|_{S} \geqslant|s|\left(|N|_{S}-4 \Delta\right)$. Thus

$$
|s| \leqslant \frac{N}{|N|_{S}-4 \Delta} .
$$

This completes the proof of Proposition 3.3.

REMARK 3.5. Note that the lemma is not true if we do not make some assumptions about the geometry of $\Gamma$. Consider, for example, the Cayley graph of $\mathbb{Z}$ with respect to the generating set $S=\left\{1,2,4, \ldots, 2^{n}, \ldots\right\}$.

Corollary 3.6. If a group $G$ quasi-acts on a tree $T$ and $g \in G$, then either $g$ quasi-acts hyperbolically or $g$ quasi-acts elliptically.

Proof. Define a quasi-action of $\mathbb{Z}$ on $T$ by $n(x)=g^{n}(x)$. By Proposition 3.1 there is some $S \subset \mathbb{Z}$ and $p \in T$ so that $\Gamma(\mathbb{Z}, S)$ embeds quasi-isometrically and coarsely equivariantly into $T$ via $n \mapsto g^{n}(p)$. By Proposition $3.3, \Gamma(\mathbb{Z}, S)$ must either have finite diameter or $S$ must be finite. If $\Gamma(\mathbb{Z}, S)$ has finite diameter, then so does the orbit $\langle g\rangle p$, and so $g$ quasi-acts elliptically. If $S$ is finite, then $t \mapsto\lfloor t\rfloor$ is a quasi-geodesic in $\Gamma(\mathbb{Z}, S)$. As $n \mapsto g^{n}(p)$ is a quasi-isometric embedding, it follows that $g$ quasi-acts hyperbolically.

\subsection{Extracting a pseudocharacter}

Lemma 3.7. Suppose $\rho: X \rightarrow \mathbb{R}$ is a $(R, \epsilon)$-quasi-isometry, where $X$ is a graph. Then there is a $\left(1, \epsilon^{\prime}\right)$-quasi-isometry $\rho^{\prime}: X \rightarrow \mathbb{R}$ for some $\epsilon^{\prime}$.

Proof. Suppose that $\rho: X \rightarrow \mathbb{R}$ is a $(R, \epsilon)$-quasi-isometry. By adjusting $\rho$ to be affine on edges and allowing $\epsilon$ to get a bit larger, we may assume that $\rho$ is continuous.

As $\rho$ is a $(R, \epsilon)$-quasi-isometry, the diameter of $\rho^{-1}(0)$ is at most $\epsilon$. Furthermore, $X \backslash \rho^{-1}(0)$ has exactly two unbounded path components, which we denote $P$ and $M$. (We may suppose that $P \subseteq \rho^{-1}(0, \infty)$ and $M \subseteq \rho^{-1}(-\infty, 0)$.) We define a new 
$\operatorname{map} \rho^{\prime}: X \rightarrow \mathbb{R}$ by

$$
\rho^{\prime}(y)= \begin{cases}d\left(y, \rho^{-1}(0)\right) & \text { if } y \in P \\ -d\left(y, \rho^{-1}(0)\right) & \text { if } y \in M \\ 0 & \text { otherwise }\end{cases}
$$

We now need to prove the following claim.

Claim 3.8. The diameter of $\rho^{-1}(c)$ for $c \in \mathbb{R}$ is at most $4 \epsilon$. The diameter of $\rho^{\prime-1}(0)$ is at most $2 \epsilon$.

Proof. First we bound the diameter of $\rho^{-1}(0)$. If $x$ and $y$ are both in $\rho^{-1}(0)$, then $d(x, y) \leqslant \epsilon$. Suppose that $p \in \rho^{\prime-1}(0) \backslash \rho^{-1}(0)$. By continuity, there is some $z \in P \cup M$ so that $\rho(z)=\rho(p)$. Any path from $z$ to $p$ must of course pass through $\rho^{-1}(0)$. Thus $d\left(p, \rho^{-1}(0)\right)<d(z, p) \leqslant \epsilon$, and so the diameter of $\rho^{\prime-1}(0)$ is at most $2 \epsilon$.

Let $c \in \mathbb{R} \backslash\{0\}$, and suppose $x$ and $y$ are in $\rho^{-1}(c)$. If $\rho(x)=\rho(y)$, then $d(x, y) \leqslant \epsilon$. Note that $x$ and $y$ are either both in $P$ or both in $M$, so the signs of $\rho(x)$ and $\rho(y)$ are the same. Suppose that $|\rho(y)|>|\rho(x)|$. Let $z \in \rho^{-1}(0)$, and let $x^{\prime}$ be a point on the geodesic from $z$ to $y$ so that $\rho\left(x^{\prime}\right)=\rho(x)$. Note that $d\left(x, x^{\prime}\right) \leqslant \epsilon$. Since $z \in \rho^{-1}(0)$ and $\rho^{\prime}(x)=\rho^{\prime}(y)$, then $|d(z, x)-d(z, y)| \leqslant 2 \epsilon$. Now, $d(x, y) \leqslant d\left(y, x^{\prime}\right)+d\left(x, x^{\prime}\right) \leqslant d\left(y, x^{\prime}\right)+\epsilon$. So we are done if we get a bound on $d\left(y, x^{\prime}\right)$. Note that $d(y, z)=d\left(y, x^{\prime}\right)+d\left(x^{\prime}, z\right) \geqslant d\left(y, x^{\prime}\right)+d(x, z)-\epsilon$. Rearranging we get $d\left(y, x^{\prime}\right) \leqslant d(y, z)-d(x, z)+\epsilon \leqslant|d(y, z)-d(x, z)| \leqslant 3 \epsilon$, and so $d(x, y) \leqslant 4 \epsilon$.

Returning to the proof of Lemma 3.7, we now show that $\rho^{\prime}$ is a $(1,5 \epsilon)$-quasiisometry. Let $x, y \in X$. By the previous claim, we may assume that $\rho^{\prime}(x) \neq \rho^{\prime}(y)$. We then have (up to switching $x$ and $y$ ) three cases to consider:

(i) $\rho^{\prime}(x)=0$;

(ii) $\rho^{\prime}(x)<0<\rho^{\prime}(y)$;

(iii) $\rho^{\prime}(y)<\rho^{\prime}(x)<0$ or $0<\rho^{\prime}(x)<\rho^{\prime}(y)$.

In case (i), $\left|\rho^{\prime}(y)-\rho^{\prime}(x)\right|=\left|\rho^{\prime}(y)\right|=d\left(y, \rho^{-1}(0)\right)$, which differs by at most $2 \epsilon$ from $d(x, y)$.

In case (ii), note that any path from $x$ to $y$ must pass through $\rho^{-1}(0)$. Let $z \in \rho^{-1}(0)$ lie on some geodesic between $x$ and $y$. We then have

$$
\left|\rho^{\prime}(y)-\rho^{\prime}(x)\right|=\left|\rho^{\prime}(x)\right|+\left|\rho^{\prime}(y)\right| \leqslant d(x, z)+d(y, z)=d(x, y) .
$$

On the other hand, since $\rho^{-1}(0)$ has diameter at most $\epsilon$,

$$
\left|\rho^{\prime}(y)-\rho^{\prime}(x)\right| \geqslant d(x, z)-\epsilon+d(y, z)-\epsilon=d(x, y)-2 \epsilon .
$$

Thus in this case $\left|\rho^{\prime}(x)-\rho^{\prime}(y)\right|$ differs from $d(x, y)$ by at most $2 \epsilon$.

In case (iii), let $z \in \rho^{-1}(0)$ be arbitrary. Then,

$$
d(x, y) \geqslant d(y, z)-d(x, z) \geqslant\left|\rho^{\prime}(y)\right|-\left|\rho^{\prime}(x)\right|-\epsilon=\left|\rho^{\prime}(y)-\rho^{\prime}(x)\right|-2 \epsilon .
$$


For the remaining inequality, let $x^{\prime}$ be a point on a geodesic between $y$ and $z$ (see Figure 8) so that $\rho^{\prime}\left(x^{\prime}\right)=\rho(x)$. Then by Claim $3.8, d\left(x, x^{\prime}\right) \leqslant 4 \epsilon$, and so

$$
\begin{aligned}
d(x, y) & \leqslant d\left(x^{\prime}, y\right)+4 \epsilon \\
& =d(y, z)-d\left(x^{\prime}, z\right)+4 \epsilon \\
& \leqslant\left|\rho^{\prime}(y)\right|+\epsilon-\left|\rho^{\prime}\left(x^{\prime}\right)\right|+4 \epsilon \\
& =\left|\rho^{\prime}(y)-\rho^{\prime}(x)\right|+5 \epsilon .
\end{aligned}
$$

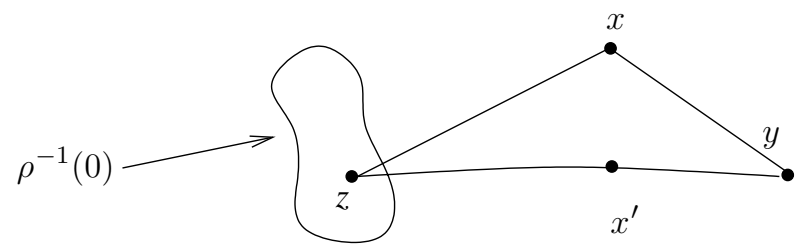

Figure 8. Case (iii): $\rho^{\prime}(x)$ and $\rho^{\prime}(y)$ have the same sign.

Combining the upper and lower bounds just obtained, we see that, in case (iii), $\left|\rho^{\prime}(x)-\rho^{\prime}(y)\right|$ cannot differ from $d(x, y)$ by more than $5 \epsilon$. In all the other cases, $\left|\rho^{\prime}(x)-\rho^{\prime}(y)\right|$ is even closer to $d(x, y)$, and so $\rho^{\prime}$ is a $(1,5 \epsilon)$-quasi-isometry.

The remainder of this section is devoted to showing that given a quasi-action of a group $G$ on a tree $T$, so that one point of $\partial T$ is fixed by $G$, we may find a pseudocharacter which is nonzero precisely on those elements of $G$ which act hyperbolically. In spirit, this pseudocharacter is what we should expect to get by picking an appropriate Busemann function and looking at its values on an orbit.

During the proof of the following proposition, we shall need to prove six claims.

Proposition 3.9. Suppose the finitely generated group $G$ quasi-acts on a simplicial tree $T$, and suppose that $G$ fixes some point $e \in \partial T$. Then there is a pseudocharacter $\chi: G \rightarrow \mathbb{R}$ so that $\chi(g)=0$ if and only if $g$ quasi-acts elliptically.

Proof. If every $g \in G$ quasi-acts elliptically, we may simply set $\chi=0$. Otherwise, Corollary 3.6 implies that some $\pi \in G$ quasi-acts hyperbolically. By possibly replacing $\pi$ with $\pi^{-1}$, we may suppose that $\lim _{n \rightarrow \infty} \pi^{n} x=e$ for any $x \in T$. Fix $p \in T$ and let $R$ be large enough so that if $S=\{g \in G \mid d(g p, p) \leqslant R\}$ then $\Gamma=\Gamma(G, S)$ embeds quasi-isometrically in $T$ as in the conclusion of Proposition 3.1. By possibly making $R$ a bit larger, we may assume that $\pi \in S$. Let $Q: \Gamma \rightarrow T$ be the quasi-isometric embedding defined by $Q(g)=g p$. This map is a quasi-isometry onto its image (a subtree of $T$ ), and so $\Gamma$ is a $\delta$-quasi-tree for some $\delta$. Moreover, the map $q$ extends to a continuous equivariant injection $\bar{Q}: \partial \Gamma \rightarrow \partial T$. The point $e$ is in the image of $\bar{Q}$, since $e=\lim _{n \rightarrow \infty} \pi^{n}(p)=\bar{Q}\left(\lim _{n \rightarrow \infty} \pi^{n}\right)$.

Since $\pi \in S, \pi^{n}$ is connected to $\pi^{n+1}$ by an edge for all $n$ and there is an obvious unit speed quasi-axis $\gamma: \mathbb{R} \rightarrow \Gamma$ for $\pi$ satisfying $\gamma(n)=\pi^{n}$. This $\gamma$ is a ( $L, D$ )-quasi-geodesic for some $L \geqslant 1$ and $D \geqslant 0$.

We will begin by finding a 'partial quasicharacter' on those group elements which are not too far from $\gamma$. We find a sequence of constants $B_{0}<B_{1}<B_{2}$ which 
ensure various kinds of good behavior on $B_{i}$-neighborhoods of $\gamma$. (Note: it will make things slightly easier if we choose the $B_{i}$ all to be positive integers, and to satisfy $B_{i+1}>10 B_{i}$. This causes no loss of generality, though of course we will not get the best possible constants.)

Since $e$ is fixed, $g \gamma([0, \infty))$ is always a finite Hausdorff distance from $\gamma([0, \infty))$. The first claim implies that it is always eventually in some fixed neighborhood of $\gamma$.

Claim 3.10. There is a constant $B_{0}$ with the following property: for any $\rho>0$, there is some $t(\rho) \geqslant 0$ so that if $d(g, \gamma) \leqslant \rho$, then $g \gamma([t(\rho), \infty))$ lies in a $B_{0^{-}}$ neighborhood of $\gamma$.

Proof. The group element $g$ fixes the point $e$ in $\partial \Gamma$ corresponding to $\lim _{t \rightarrow \infty}$ $\gamma(t)$. In particular, $\lim _{t \rightarrow \infty} g \gamma(t)=e$. Note that $Q \circ \gamma$ is a continuous $(K L, K D+C)$ quasi-geodesic in the tree $T$, and so there is some geodesic $\sigma_{1}: \mathbb{R} \rightarrow T$ whose image is contained in the image of $Q \circ \gamma$ and which has the same endpoints in $\partial T$ as does $Q \circ \gamma$. We parameterize $\sigma_{1}$ so that $\sigma_{1}(t)$ tends toward $e$ as $t$ tends to positive infinity. There is also a geodesic ray $\sigma_{2}:[0, \infty) \rightarrow T$ whose image is contained in the image of $\left.Q \circ g \gamma\right|_{[0, \infty)}$ and which has the same endpoints in $T \cup \partial T$ as does $\left.Q \circ g \gamma\right|_{[0, \infty)}$. Let $\sigma:[0, \infty) \rightarrow T$ be the unique geodesic ray whose image is the intersection of the images of $\sigma_{1}$ and $\sigma_{2}$.

Let $t_{1}=\inf \{t \mid Q \circ \gamma(t)=\sigma(0)\}$ and $t_{g}=\inf \{t \mid Q \circ g \gamma(t)=\sigma(0)\}$. Since $\left.Q \circ \gamma\right|_{\left[t_{1}, \infty\right)}$ and $\left.Q \circ g \gamma\right|_{\left[t_{g}, \infty\right)}$ are $(K L, K D+C)$-quasi-geodesic rays with the same endpoints as $\sigma$, neither can stray from a $B:=B(K L, K D+C, 0)$-neighborhood of $\sigma$ (Lemma 2.11 applied in the case of $T$, a 0 -hyperbolic space). Thus for $t \geqslant t_{g}$, the distance between $g \gamma(t)$ and $\gamma$ is at most $K(B+C)$. (This is because $Q$ is a $(K, C)$-quasi-isometry and the image of $Q \circ \gamma$ includes all of $\sigma$.)

Fixing $B_{0}=K B+K C$, it remains only to show that $t_{g}$ can be bounded by some function of $\rho=d(g, \gamma)$. First note that $d(Q(g), Q(\gamma)) \leqslant K \rho+C$. Since $\sigma(0)$ is the closest point on $\sigma_{1}$ to $Q(g)$, and since $Q \circ \gamma$ lies in a $B$-neighborhood of $\sigma_{1}$, we have $d(Q(g), \sigma(0)) \leqslant d(Q(g), Q(\gamma))+B$, and so $d(Q(g), \sigma(0)) \leqslant K \rho+C+B$. Finally, since $d(Q(g), \sigma(0))=d\left(Q \circ g \gamma(0), Q \circ g \gamma\left(t_{g}\right)\right)$ and $Q \circ g \gamma$ is a $(K L, K D+C)$-quasigeodesic, we have

$$
\frac{1}{K L} t_{g}-(K D+C) \leqslant d(Q(g), \sigma(0)) .
$$

We may therefore set $t(\rho)=K L(K \rho+C+B+K D+C)$ and the claim is established.

Let $N_{0}=\left\{x \in \Gamma \mid d(x, \gamma) \leqslant B_{0}\right\}$, and let $G_{0}=G \cap N_{0}$.

Claim 3.11. There is some $B_{1}$ so that, if $g \in G_{0}$, then $\left.g \gamma\right|_{[0, \infty)}$ is within $B_{1}$ of $\gamma$.

Proof. By Claim 3.10, there is some $t=t\left(B_{0}\right)$ so that, if $g$ is within $B_{0}$ of $\gamma$, then $\left.g \gamma\right|_{[t, \infty)}$ is within $B_{0}$ of $\gamma$. Thus we need only worry about the initial segment $\left.g \gamma\right|_{[0, t]}$. Since $g \gamma$ is always an $(L, D)$-quasi-geodesic, it certainly cannot go further away than $L t+D$ in this time, and so any $B_{1} \geqslant B_{0}+L t+D$ will suffice.

(As mentioned before, we want to make sure we have a healthy gap between $B_{0}$ and $B_{1}$, so we choose $B_{1}>\max \left\{B_{0}+L t+D, 10 B_{0}\right\}$.) 
Let $N_{1}=\left\{x \in \Gamma \mid d(x, \gamma) \leqslant B_{1}\right\}$, with the metric induced by inclusion in $\Gamma$. Notice that this metric may not be the same as the path metric on $N_{1}$, so $N_{1}$ might not be a geodesic metric space. We can however find a bigger neighborhood in which geodesics between different points of $N_{1}$ may always be found.

Claim 3.12. There is some $B_{2}$ so that, if $N_{2}=\left\{x \in \Gamma \mid d(x, \gamma) \leqslant B_{2}\right\}$ is given the path metric, then the inclusion of $N_{1}$ in $N_{2}$ is an isometric embedding. That is, between any two points of $N_{1}$ there is a geodesic in $\Gamma$ between them which lies in a $B_{2}$-neighborhood of $\gamma$.

Proof. Let $x_{1}$ and $x_{2}$ lie in $N_{1}$, and let $t_{1}, t_{2}$ be real numbers so that $d\left(x_{i}, \gamma\left(t_{i}\right)\right)$ $\leqslant B_{1}$ for $i \in\{1,2\}$. Since $\gamma$ is an $(L, D)$-quasi-geodesic, we may use it to define a (not continuous) $\left(L, D+2 B_{1}\right)$-quasi-geodesic $\alpha$ from $x_{1}$ to $x_{2}$ inside $N_{1}$ as follows:

$$
\alpha(t):= \begin{cases}x_{i} & t=t_{i} \\ \gamma(t) & \text { otherwise. }\end{cases}
$$

Recall that $\Gamma$ is $\delta$-hyperbolic, and so we may make use of Lemma 2.11; any geodesic in $\Gamma$ between $x_{1}$ and $x_{2}$ lies in a $B\left(L, D+2 B_{1}, \delta\right)$-neighborhood of $\alpha$. Since $\alpha$ lies entirely inside a $B_{1}$-neighborhood of $\gamma$, we may set $B_{2}$ to some integer at least as large as $\max \left\{10 B_{1}, B_{1}+B\left(L, D+2 B_{1}, \delta\right)\right\}$. The claim is established.

\section{Claim 3.13. $\quad N_{2}$ is quasi-isometric to $\mathbb{R}$.}

Proof. By Claim 3.12, any two points in the image of $\gamma$ have distance in $N_{2}$ equal to their distance in $\Gamma$. Thus $\gamma: \mathbb{R} \rightarrow N_{2}$ is still an $(L, D)$-quasi-geodesic from the point of view of the path metric on $\mathrm{N}_{2}$.

Since every point in $N_{2}$ is within $B_{2}$ of the image of $\gamma$, the quasi-isometric embedding $\gamma: \mathbb{R} \rightarrow N_{2}$ is a quasi-isometry.

Since $B_{2}$ is assumed to be an integer, $N_{2}$ is a graph, and so Lemma 3.7 implies that for some $\epsilon>0$ there is a continuous $(1, \epsilon)$-quasi-isometry $\chi_{0}: N_{2} \rightarrow \mathbb{R}$. By composing with an isometry of $\mathbb{R}$, we may assume that $\chi_{0}(1)=0$ and that $\chi_{0}\left(\pi^{n}\right) \rightarrow$ $\infty$ as $n \rightarrow \infty$. Note that at least up to some additive error independent of $g,\left|\chi_{0}(g)\right|$ is the distance from 1 to $g$ in $\Gamma$. We will eventually show that $\chi_{0}$ is a "partial quasicharacter' on $G_{0}$ (Claim 3.15).

If $g \in G_{0}$, and $\chi_{0}(h g)-\chi_{0}(h)$ has the same sign as $\chi_{0}(g)$ whenever $h g$ and $h$ are also in $G_{0}$, we will say that $g$ is irreversible.

Claim 3.14. There is some $R$ so that, if $g \in G_{0}$ and $\left|\chi_{0}(g)\right|>R$, then $g$ is irreversible.

Proof. We prove the claim for $R=(\epsilon+D) L^{2}+D+B_{0}$. Assume that $g \in G_{0}$ is not irreversible. That is, suppose that there is some $h \in G_{0}$ so that $g h \in G_{0}$ but the signs of $\chi_{0}(g)$ and $\chi_{0}(h g)-\chi_{0}(h)$ differ. Since $g \in G_{0}$ there is some point $\pi^{m}$ on $\gamma$ so that $d\left(g, \pi^{m}\right) \leqslant B_{0}$.

Suppose the sign of $\chi_{0}\left(\pi^{m}\right)$ differs from the sign of $\chi_{0}(g)$. Since $\pi^{m}$ and $g$ are both inside $N_{1}$, a geodesic between $\pi^{m}$ and $g$ lies in $N_{2}$. By continuity there is some point 
$w$ on this geodesic with $\chi_{0}(w)=0$. Thus $d(g, 1) \leqslant d(g, w)+d(w, 1) \leqslant B_{0}+\epsilon \leqslant R$. Thus we may suppose that the signs of $\chi_{0}\left(\pi^{m}\right)$ and $\chi_{0}(g)$ agree.

Suppose that the sign of $m$ differs from the signs of $\chi_{0}\left(\pi^{m}\right)$ and $\chi_{0}(g)$. In this case there is some $t \in \mathbb{R}$ having the same sign as $m$, with $|t|>|m|$, but with $\chi_{0}(\gamma(t))=0$. Since $\chi_{0}(\gamma(t))=0=\chi_{0}(1)$, we must have $d(\gamma(t), 1) \leqslant \epsilon$, which leads to the following:

$$
\begin{gathered}
\frac{1}{L}|t|-D \leqslant \epsilon \Rightarrow|t| \leqslant L(\epsilon+D) \Rightarrow|m| \leqslant L(\epsilon+D) \\
\Rightarrow d\left(1, \pi^{m}\right) \leqslant L^{2}(\epsilon+D)+D \Rightarrow d(g, 1) \leqslant L^{2}(\epsilon+D)+D+B_{0}=R .
\end{gathered}
$$

We may therefore suppose that the signs of $m, \chi_{0}\left(\pi^{m}\right)$ and $\chi_{0}(g)$ all agree.

We next turn our attention to the order of $\chi_{0}(h), \chi_{0}\left(h \pi^{m}\right)$, and $\chi_{0}(h g)$. Suppose first that $\chi_{0}(h)$ is between $\chi_{0}\left(h \pi^{m}\right)$ and $\chi_{0}(h g)$. Since $h \pi^{m}$ is at most $B_{0}$ from $h g$, and $h g$ is assumed to be at most $B_{0}$ from $\gamma$, the point $h \pi^{m}$ is at most $2 B_{0}<B_{1}$ from $\gamma$. By Claim 3.12, there is a geodesic in $\Gamma$ from $h \pi^{m}$ to $h g$ entirely inside $N_{2}$. By continuity there is a point $w$ on this geodesic with $\chi_{0}(w)=\chi_{0}(h)$. As $\chi_{0}$ is a $(1, \epsilon)$-quasi-isometry, $d(w, h) \leqslant \epsilon$, and so

$$
d(g, 1)=d(h g, h) \leqslant d(h g, w)+\epsilon \leqslant d\left(h g, h \pi^{m}\right)+\epsilon \leqslant B_{0}+\epsilon
$$

establishes the claim in this case. We may therefore assume that $\chi_{0}(h)$ is not between $\chi_{0}\left(h \pi^{m}\right)$ and $\chi_{0}(h g)$.

It is now necessary to distinguish cases according to the sign of $\chi_{0}(g)$. We first deal with $\chi_{0}(g)$ negative and $\chi_{0}(h g)-\chi_{0}(h)$ positive. Together with our previous assumptions this gives $\chi_{0}\left(h \pi^{m}\right)>\chi_{0}(h)$. Since $(h \gamma)^{-1}\left(h \pi^{m}\right)=m$ is negative, $h \gamma$ must 'double back' as in Figure 9 and there must be some $s>0$ with $\chi_{0}(h \gamma(s))=$ $\chi_{0}\left(h \pi^{m}\right)$. Since $\chi_{0}$ is a $(1, \epsilon)$-quasi-isometry we have $d\left(h \gamma(s), h \pi^{m}\right) \leqslant \epsilon$, and a by now familiar looking calculation shows that $|s| \leqslant L(\epsilon+D)$ and so $d(g, 1)=$ $d(h g, h) \leqslant L^{2}(\epsilon+D)+D+B_{0}$.

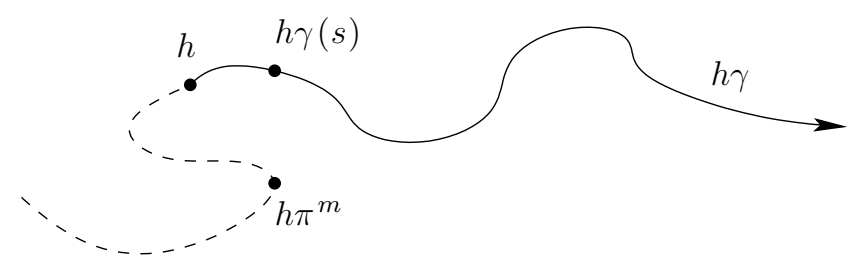

Figure 9. The case of $\chi_{0}(g)$ negative. The function $\chi_{0}$ increases to the right. Note that the dotted part of $h \gamma$ may not lie entirely in $N_{2}$, though $h \pi^{m}$ is in $N_{2}$.

In case $\chi_{0}(g)$ and $m$ are positive but $\chi_{0}(h g)-\chi_{0}(h)$ is negative, we again deduce that $h \gamma$ must double back, but now the picture is slightly different and shown in Figure 10. Continuity ensures the existence of some $s>m>0$ on $\gamma$ so that $\chi_{0}(h \gamma(s))=\chi_{0}(h)$ (and hence $\left.d(h \gamma(s), h) \leqslant \epsilon\right)$. Now the reader may verify that the bound $s-m \leqslant s \leqslant L(\epsilon+D)$ leads to $d(g, 1) \leqslant L^{2}(\epsilon+D)+D+B_{0}$ in this final case. This completes the proof of Claim 3.14.

However, this is finally enough to prove that $\chi_{0}$ is a partial quasicharacter. 


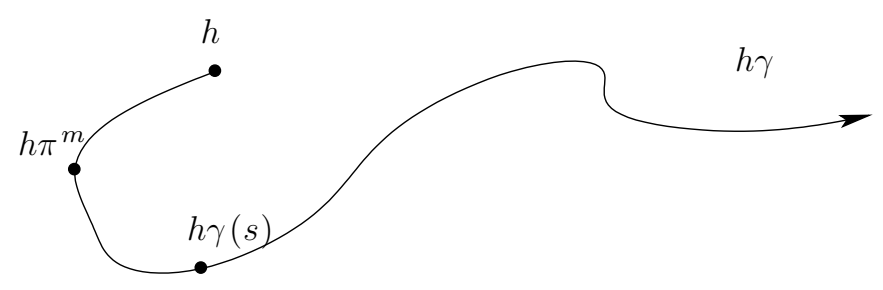

Figure 10. The case of $\chi_{0}(g)$ positive.

Claim 3.15. There is some number $\Delta_{0}$ so that, if $g, h$, and $g h$ all lie in $G_{0}$, then $\left|\chi_{0}(g h)-\chi_{0}(g)-\chi_{0}(h)\right| \leqslant \Delta_{0}$.

Proof. Suppose $g, h$, and $g h$ all lie in $G_{0}$. If $h$ is not irreversible, then $\left|\chi_{0}(h)\right| \leqslant R$ by Claim 3.14, and so $d(g h, g)=d(h, 1) \leqslant R+\epsilon$. Thus since $\chi_{0}$ is a $(1, \epsilon)$-quasiisometry, $\left|\chi_{0}(g h)-\chi_{0}(g)\right| \leqslant R+2 \epsilon$ and $\left|\chi_{0}(g h)-\chi_{0}(g)-\chi_{0}(h)\right| \leqslant 2 R+2 \epsilon$ in this case.

Suppose that $h$ is irreversible. Then the sign of $\chi_{0}(g h)-\chi_{0}(g)$ is the same as the sign of $\chi_{0}(h)$. Since $d(g h, g)=d(h, 1)$, the magnitude $\left|\chi_{0}(g h)-\chi_{0}(g)\right|$ is within $2 \epsilon$ of $\left|\chi_{0}(h)\right|$. Thus $\left|\chi_{0}(g h)-\chi_{0}(g)-\chi_{0}(h)\right| \leqslant 2 \epsilon$. In both cases $\left|\chi_{0}(g h)-\chi_{0}(g)-\chi_{0}(h)\right|$ is bounded above by $2 R+2 \epsilon$, and so $\chi_{0}$ is a partial quasicharacter with defect $\Delta_{0} \leqslant 2 R+2 \epsilon$.

We now can prove Proposition 3.9 by 'extending' $\chi_{0}$ to all of $G$. For $i>0$, let $G_{i}=\left\{g \in G \mid \pi^{-i} g \pi^{i} \in G_{0}\right\}$. We claim that any $g \in G$ is contained in all but finitely many $G_{i}$. Indeed, we have already shown (Claim 3.10) that $g \pi^{k}$ is always in $G_{0}$ for large enough $k$. Since $\pi G_{0}=G_{0}$, then $\pi^{-k} g \pi^{k}$ is in all $G_{i}$ for all such $k$. Thus if we can consistently (at least up to a bounded error) define partial quasicharacters on all the $G_{i}$, we will have defined one on $G$. Let $\chi_{i}: G_{i} \rightarrow \mathbb{R}$ be defined by $\chi_{i}(g)=\chi_{0}\left(\pi^{-i} g \pi^{i}\right)$, and define $\chi(g)=\lim \sup _{i \rightarrow \infty} \chi_{i}(g)$. (We could just as well use the lim inf or any number between the two; the lim inf and limsup will be seen to be boundedly different from one another, and we only need to understand the coarse properties of $\chi$.)

We now bound the error $|\delta \chi(g, h)|=|\chi(g h)-\chi(g)-\chi(h)|$ for $g, h \in G$. Note that if $\chi_{i}(g)$ and $\chi_{j}(g)$ are both defined for $i<j$, then

$$
\left|\chi_{i}(g)-\chi_{j}(g)\right|=\left|\chi_{0}\left(\pi^{-i} g \pi^{i}\right)-\chi_{0}\left(\left(\pi^{i-j}\right)\left(\pi^{-i} g \pi^{i}\right)\left(\pi^{j-i}\right)\right)\right| \leqslant 3 \Delta_{0}
$$

since all powers of $\pi$ also lie in $G_{0}$. Thus $\chi(g)-\chi_{i}(g)$ is also at most $3 \Delta_{0}$, whenever $\chi_{i}(g)$ is defined. Let $k$ be any number so that $g, h$, and $g h$ all lie in $G_{k}$. Then $|\delta \chi(g, h)| \leqslant\left|\chi_{k}(g h)-\chi_{k}(g)-\chi_{k}(h)\right|+9 \Delta_{0}$. Finally,

$$
\left|\chi_{k}(g h)-\chi_{k}(g)-\chi_{k}(h)\right|=\left|\chi_{0}\left(\pi^{-k} g h \pi^{k}\right)-\chi_{0}\left(\pi^{-k} g \pi^{k}\right)-\chi_{0}\left(\pi^{-k} h \pi^{k}\right)\right| \leqslant \Delta_{0}
$$

implies that $|\delta \chi(g, h)| \leqslant 10 \Delta_{0}$, and so $\chi$ is a quasicharacter. As in Remark 2.20, if we define

$$
\bar{\chi}(g)=\lim _{n \rightarrow \infty} \frac{\chi\left(g^{n}\right)}{n},
$$

then $\bar{\chi}$ is a pseudocharacter which is boundedly different from $\chi$. 
If $g$ is elliptic, then $\chi\left(g^{n}\right)$ will be bounded independently of $n$, and so $\bar{\chi}(g)=0$.

Finally, suppose that $g$ is hyperbolic. Then either $\left\{g^{n}\right\}$ or $\left\{g^{-n}\right\}$ tend toward a point fixed by $G$ in $\partial \Gamma$. By replacing $g$ by $g^{-1}$ if necessary, we may assume that the $g^{n}$ tend toward the fixed point as $n \rightarrow \infty$.

We now show that the $g^{n}$ for $n>0$ all lie in some fixed $G_{k}$. We construct a quasiaxis for $g$ by setting $\left.\alpha\right|_{[0, d(1, g)]}$ to be some geodesic between 1 and $g$ and requiring that $\alpha(t+d(1, g))=g \alpha(t)$. The restriction $\alpha_{[0, \infty)}$ is an $\left(L_{g}, D_{g}\right)$-quasi-geodesic starting at 1 and limiting on $e$ just as $\gamma_{[0, \infty)}$ is. By Lemma 2.11 it therefore stays in some bounded $B_{g}:=B\left(\max \left\{L, L_{g}\right\}, \max \left\{D, D_{g}\right\}, \delta\right)$-neighborhood of $\gamma$. Together with Claim 3.10, this implies that there is some $N$ so that $g^{n} \pi^{k} \in G_{0}$ for all $n>0$, $k>N$. Thus all positive powers of $g$ lie in $G_{N}$ for some fixed $N$ which depends only on $g$. Since $g$ acts hyperbolically, $d\left(1, \pi^{-N} g^{n} \pi^{N}\right)$ tends to infinity as $n$ tends to infinity, implying that $\chi_{N}$ is unbounded on $\left\{g^{n} \mid n>0\right\}$. This implies that $\chi$ and $\bar{\chi}$ are likewise unbounded on $\left\{g^{n} \mid n>0\right\}$. Since $\bar{\chi}$ is a homomorphism on cyclic subgroups, we must have $\bar{\chi}(g) \neq 0$. This completes the proof of Proposition 3.9.

\section{A class of groups with Property (QFA)}

In this section we give an example of how the results of Section 3 can be used to establish Property (QFA).

Definition 4.1. Let $G$ be a group, and let $g$ be an element of $G$. We will say that $g$ is a stubborn element of $G$ if, for all $H<G$ with $[G: H] \leqslant 2$, there exists some integer $k_{H}>0$ so that $g^{k_{H}} \in[H, H]$.

LEMma 4.2. Let $B$ be an amenable group, quasi-acting on a tree $T$. If $b$ is a stubborn element of $B$, then $b$ quasi-acts elliptically.

Proof. Suppose that $b$ does not quasi-act elliptically. We show that $B$ cannot be amenable. By Corollary 3.6, $b$ quasi-acts hyperbolically, and therefore fixes exactly two points in $\partial T$. Let $\operatorname{Fix}(b)=\left\{e_{1}, e_{2}\right\}$ be the fixed point set of $b$.

We claim that either $B$ preserves $\operatorname{Fix}(b)$ or fixes either $e_{1}$ or $e_{2}$. Indeed, suppose that $\operatorname{Fix}(b)$ is not preserved by all of $B$. If there are elements $b_{1}$ and $b_{2}$ so that $b_{1}\left(e_{1}\right) \neq e_{1}$ and $b_{2}\left(e_{2}\right) \neq e_{2}$, then a simple ping-pong argument shows that $B$ must contain a nonabelian free subgroup and thus cannot be amenable.

We may therefore assume that there is some subgroup $H \leqslant B$ of index at most 2 which fixes some point in $\partial X$ and contains the element $b$. By Proposition 3.9 there is a pseudocharacter $p: H \rightarrow \mathbb{R}$ so that $p(b) \neq 0$. Since $b^{k_{H}} \in[H, H], p$ is not a homomorphism, and so $[\delta p]$ is a nonzero element of $H_{b}^{2}(H, \mathbb{R})$. Theorem 2.21 then implies that $H$ is not amenable, and so $B$ is not amenable.

Definition 4.3. A group $G$ is boundedly generated if there exists a finite tuple $\left(g_{1}, \ldots, g_{n}\right)$ of elements of $G$ so that any $g \in G$ is equal to $g_{1}^{\alpha_{1}} \cdots g_{n}^{\alpha_{n}}$ for some tuple $\left(\alpha_{1}, \ldots, \alpha_{n}\right)$ of integers. This is easily seen to be equivalent to the following condition: there is some finite set $\left\{g_{1}, \ldots, g_{n}\right\} \subset G$ such that the Cayley graph $\Gamma(G, S)$ has finite diameter for any $S \supseteq\left\{g_{i}^{m} \mid i \in\{1, \ldots, n\}, m \in \mathbb{Z}\right\}$. In either case we say that $G$ is boundedly generated by the elements $g_{1}, \ldots, g_{n}$. 
THEOREM 4.4. Let $G$ be a group which is boundedly generated by elements $g_{1}, \ldots, g_{n}$, so that, for each $i, g_{i}$ is a stubborn element of $B_{i}$ for some $B_{i}<G$. Then $G$ has Property (QFA).

Proof. Suppose that $G$ quasi-acts on some tree $T$. By Lemma 4.2, each $g_{i}$ quasiacts elliptically.

Fix $p \in T$. We must show that the orbit $G p$ is bounded. Let

$$
R>\sup \left\{d\left(p, g_{i}^{m} p\right) \mid i \in\{1, \ldots, n\}, m \in \mathbb{Z}\right\} .
$$

Since each $g_{i}$ has bounded orbits, we may find such a finite $R$. By perhaps increasing $R$, we may assume that $R$ is large enough that $\Gamma(G, S)$ coarsely equivariantly quasi-isometrically embeds in $T$, for $S=\{g \in G \mid d(g p, p) \leqslant R\}$ (applying Proposition 3.1). However, since $S \supseteq\left\{g_{i}^{m} \mid i \in\{1, \ldots, n\}, m \in \mathbb{Z}\right\}$, the Cayley graph $\Gamma(G, S)$ has finite diameter. It follows that the orbit $G p$ has finite diameter.

Corollary 4.5. Let $K$ be a number field, and $\mathcal{O}$ its ring of integers. Then $\mathrm{SL}(n, \mathcal{O})$ has Property (QFA), for $n>2$. In particular, $\operatorname{SL}(n, \mathbb{Z})$ has Property (QFA), for $n>2$.

Proof. Let $\Lambda=\left\{\lambda_{1}, \ldots, \lambda_{k}\right\}$ be an integral basis for $\mathcal{O}$ (for $\mathcal{O}=\mathbb{Z}, \Lambda=\{1\}$ ). Let $e_{i j}^{\lambda}$ be the matrix equal to the identity matrix except for the $i j$ th entry, which is equal to $\lambda$. Note that $\left(e_{i j}^{\lambda}\right)^{-1}=e_{i j}^{-\lambda}$. It is shown in $[6]$ that $\operatorname{SL}(n, \mathcal{O})$ is boundedly generated by $\left\{e_{i j}^{\lambda} \mid i \neq j, \lambda \in \Lambda\right\}$. It can be easily verified that $e_{i k}^{\lambda} e_{k j}^{1} e_{i k}^{-\lambda} e_{k j}^{-1}=e_{i j}^{\lambda}$, provided that $i, j$ and $k$ are all distinct. Furthermore, the subgroup $B_{i j}=\left\langle e_{i k}^{\lambda}, e_{k j}^{1}\right\rangle$ is nilpotent and therefore amenable. If $H<B_{i j}$ is a subgroup of index 2, note that $e_{i k}^{2 \lambda}$ and $e_{k j}^{2}$ are contained in $H$. The commutator $\left[e_{i k}^{2 \lambda}, e_{k j}^{2}\right]=e_{i j}^{4 \lambda}=\left(e_{i j}^{\lambda}\right)^{4}$, so $e_{i j}^{\lambda}$ is stubborn. Theorem 4.4 can then be applied.

REMARK 4.6. It is natural to wonder to what extent the hypotheses of Theorem 4.4 can be weakened or modified, and what other groups can be shown to have Property (QFA) using these methods. Using Theorem 4.4 together with theorems of Tits [23] and Tavgen' [22] it is possible to prove that Property (QFA) holds for simple, simply connected Chevalley groups of semisimple rank at least two over the ring of integers of a number field.

One might wonder whether bounded generation plus (FA) is enough to guarantee (QFA). In fact there are groups (certain central extensions of lattices in higher rank Lie groups) which are boundedly generated and have Property (FA) (in fact they are Kazhdan, which is stronger), but which admit a nontrivial pseudocharacter. Thus, by the construction in Example 2.14 these groups do not have Property (QFA). These examples are described in the Appendix.

\section{5. (QFA)-type properties for particular kinds of trees}

We can break Question 1.1 down into parts by considering only cobounded actions on particular types of trees. That is the strategy in this section. 
Definition 5.1. If $\mathcal{T}$ is a class of (infinite diameter) trees, and $G$ does not admit a cocompact action on any tree in $\mathcal{T}$, then we will say that $G$ has Property $(\mathrm{FA} \mathcal{T})$. If $G$ does not admit a cobounded quasi-action on any tree in $\mathcal{T}$, we will say that $G$ has Property $(\mathrm{QFA} \mathcal{T})$.

Note that while $(\mathrm{QFA} \mathcal{T})$ always implies $(\mathrm{FA} \mathcal{T}),(\mathrm{FA} \mathcal{T})$ does not usually imply $(\mathrm{QFAT})$.

Definition 5.2. A tree $T$ is bushy if there is a number $B$, called the bushiness constant of $T$, so that for any point $p \in T$ the set $\{x \in T \mid d(p, x) \geqslant B\}$ has at least three components. We will say that $T$ is finitely bushy if in addition $\{x \in$ $T \mid d(p, x) \geqslant B\}$ always has finitely many components. If $B$ can be chosen so that $\{x \in T \mid d(p, x) \geqslant B\}$ has a countable infinity of components for every $x \in T$, we say the tree is countably bushy.

We write $\mathcal{B}$ for the set of finitely bushy trees and $\mathcal{C}$ for the set of countably bushy trees.

The proof of the following proposition is left to the reader.

Proposition 5.3. A finitely generated group $G$ has Property (QFA) if and only if it has all three of the properties $(\mathrm{QFA}\{\mathbb{R}\}),(\mathrm{QFA} \mathcal{B})$ and $(\mathrm{QFAC})$.

EXAMPLE 5.4. The group $\mathbb{Z}$ obviously does not have (QFA $\{\mathbb{R}\}$ ), but Propositions 3.1 and 3.3 imply that $\mathbb{Z}$ has $(\mathrm{QFA} \mathcal{B})$ and $(\mathrm{QFAC})$.

It is a corollary of the main theorem of $[\mathbf{1 8}]$ that Properties $(\mathrm{FAB})$ and $(\mathrm{QFA} \mathcal{B})$ are equivalent. A characterization of $(\mathrm{QFA}\{\mathbb{R}\})$ is given by Proposition 5.5 below.

Proposition 5.5. Let $G$ be a group. The following are equivalent:

(i) $G$ has Property $(\mathrm{QFA}\{\mathbb{R}\})$.

(ii) Neither $G$ nor any index 2 subgroup of $G$ has a nontrivial pseudocharacter.

Proof. We split the proof into two parts.

(i) implies (ii): we argue by assuming that (ii) does not hold and then producing a quasi-action by $G$ on $\mathbb{R}$.

Suppose either $G$ or some index subgroup of $G$ admits a nontrivial pseudocharacter. The easiest case to deal with is if $f: G \rightarrow \mathbb{R}$ is a nontrivial pseudocharacter. Then $A: G \times \mathbb{R} \rightarrow \mathbb{R}$ defined by $A(g, x)=f(g)+x$ is a cobounded quasi-action on $\mathbb{R}$.

Let $H$ be an index 2 subgroup of $G$ and suppose that $f: H \rightarrow \mathbb{R}$ is a nontrivial pseudocharacter. Fix some element $t$ of $G \backslash H$ and define $f_{t}: H \rightarrow G$ by $f_{t}(h)=$ $f(h)-f\left(t^{-1} h t\right)$. Note that $f_{t}\left(t^{-1} h t\right)=-f_{t}(h)$. There are two cases, depending on whether $f_{t}(h)=0$ for every $h$ in $H$.

If $f_{t}=0$ on $H$ we can extend $f$ to a quasicharacter $\bar{f}: G \rightarrow \mathbb{R}$ by setting

$$
\bar{f}(g)= \begin{cases}f(g) & \text { if } g \in H \\ f(h) & \text { if } g=t h \text { for } h \in H\end{cases}
$$


To see that $\bar{f}$ is a quasicharacter, we must bound $|\delta \bar{f}(\alpha, \beta)|$ in four cases, depending on whether $\alpha$ and $\beta$ are in $H$ or $t H$. In three cases, the reader may verify that $|\delta \bar{f}(\alpha, \beta)|$ is bounded by $\|\delta f\|$. In the fourth case, where $\alpha$ and $\beta$ both lie in $t H$, suppose that $\alpha=t h_{1}$ and $\beta=t h_{2}$. Then $\bar{f}(\alpha \beta)=\bar{f}\left(t h_{1} t h_{2}\right)=\bar{f}\left(t^{2}\left(t^{-1} h_{1} t\right) h_{2}\right)$, which differs from $\bar{f}(\alpha)+\bar{f} \beta$ by at most $2\|\delta f\|+\left|f\left(t^{2}\right)\right|$. Thus we have produced an unbounded quasicharacter on $G$, which may be modified to a pseudocharacter as in Remark 2.20, and we are back in the easy case.

If $f_{t}$ is not identically zero, then it is a nontrivial pseudocharacter on $H$. It is then possible to build a quasi-action of $G$ on $\mathbb{R}$ so that $t$ acts as a reflection. Namely, we set

$$
A(g, x)= \begin{cases}f_{t}(g)+x & \text { if } g \in H \\ -f_{t}(h)-x & \text { if } g=t h \text { for } h \in H .\end{cases}
$$

Each group element acts as an isometry, so we only need to show that $(\alpha)(\beta x)$ and $(\alpha \beta) x$ are uniformly close. Again there are four different cases, depending on whether $\alpha$ and $\beta$ lie in $H$ or $t H$. All four cases may safely be left to the diligent reader. Since $f$ is assumed to be nontrivial, it is unbounded on $H$, and thus this quasi-action is cobounded.

(ii) implies (i): we assume that there is a cobounded quasi-action of $G$ on $\mathbb{R}$ and produce a pseudocharacter.

Any element of $G$ must either switch $\pm \infty$ or preserve them. Thus either $G$ or an index 2 subgroup of $G$ fixes $\pm \infty$. We may then apply Proposition 3.9 to obtain a nontrivial pseudocharacter on the subgroup of $G$ fixing $\pm \infty$.

Of course it remains to give a satisfactory account of (QFAC).

Acknowledgements. Thanks are due to my doctoral advisor Daryl Cooper for asking me (more than once!) whether $\mathrm{SL}(3, \mathbb{Z})$ could quasi-act nontrivially on a tree. Thanks also go to the Oxford Mathematical Institute for hospitality while part of this work was being done. Many people have had helpful things to say to me about these problems, including Nicolas Monod, Kevin Whyte, and Hee Oh. This work was partially supported by an NSF Postdoctoral Research Fellowship and by a UCSB Graduate Division Dissertation Fellowship.

Some of these results have been worked out independently but not published by Lee Mosher, Michah Sageev and Kevin Whyte.

APPENDIX. BoundedLy GENERATED Groups WITH PSEUdocharaCters

N. Monod and B. Rémy

The aim of this appendix is to construct concrete groups which simultaneously:

(1) are boundedly generated;

(2) have Kazhdan's property (T);

(3) have a one-dimensional space of pseudocharacters. 
By (3), such groups do not have property (QFA), whilst they have property (FA) by (2); moreover the quasimorphisms in (3) cannot be bushy in the sense of [16]. Property (3) has its own interest, as all previous constructions yield infinitedimensional spaces. (By taking direct products of our examples, one gets any finite dimension.) The examples will be lattices $\widetilde{\Gamma}$ in nonlinear simple Lie groups; more precisely, starting with certain higher rank Lie groups $H$ with $\pi_{1}(H)=\mathbb{Z}$ and suitable lattices $\Gamma<H$, the group $\widetilde{\Gamma}$ will be the preimage of $\Gamma$ in the universal covering central extension

$$
0 \rightarrow \mathbb{Z} \rightarrow \widetilde{H} \rightarrow H \rightarrow 1
$$

Let us first start with any group $\Gamma$ satisfying the following cohomological properties (we refer to [5] for our use of bounded cohomology):

$\left(3^{\prime}\right)$ the second bounded cohomology $\mathrm{H}_{\mathrm{b}}^{2}(\Gamma, \mathbb{R})$ has dimension one;

$\left(3^{\prime \prime}\right)$ the natural map $\psi_{\Gamma}: \mathrm{H}_{\mathrm{b}}^{2}(\Gamma, \mathbb{R}) \rightarrow \mathrm{H}^{2}(\Gamma, \mathbb{R})$ is injective;

$\left(3^{\prime \prime \prime}\right)$ the image of the natural map $i_{\Gamma}: \mathrm{H}^{2}(\Gamma, \mathbb{Z}) \rightarrow \mathrm{H}^{2}(\Gamma, \mathbb{R})$ spans the image of $\psi_{\Gamma}$.

Claim. Under these assumptions, there is a central extension $0 \rightarrow \mathbb{Z} \rightarrow \widetilde{\Gamma} \rightarrow$ $\Gamma \rightarrow 1$ such that the kernel of $\psi_{\widetilde{\Gamma}}: \mathrm{H}_{\mathrm{b}}^{2}(\widetilde{\Gamma}, \mathbb{R}) \rightarrow \mathrm{H}^{2}(\widetilde{\Gamma}, \mathbb{R})$ has dimension one.

Proof. By the assumptions, there is $\omega_{\mathbb{Z}} \in \mathrm{H}^{2}(\Gamma, \mathbb{Z})$ and $\omega \in \mathrm{H}_{\mathrm{b}}^{2}(\Gamma, \mathbb{R})$ such that $\psi_{\Gamma}(\omega)=i_{\Gamma}\left(\omega_{\mathbb{Z}}\right) \neq 0$. The central extension $0 \rightarrow \mathbb{Z} \rightarrow \widetilde{\Gamma} \stackrel{\pi}{\longrightarrow} \Gamma \rightarrow 1$ associated to $\omega_{\mathbb{Z}}$ yields a commutative diagram:

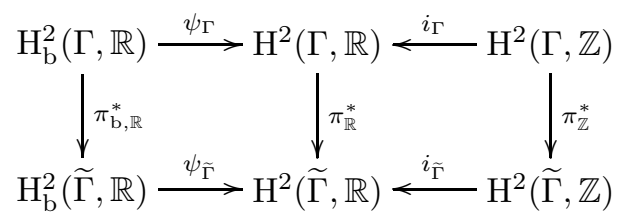

Since $\mathbb{Z}$ is amenable, $\pi_{\mathrm{b}, \mathbb{R}}^{*}$ is an isomorphism $[\mathbf{1 5}, 3.8 .4]$ (this is not true in general for $\mathbb{Z}$ coefficients). Setting $\beta:=\pi_{b, \mathbb{R}}^{*}(\omega)$, we are reduced to seeing that $\mathrm{H}_{\mathrm{b}}^{2}(\widetilde{\Gamma}, \mathbb{R})=$ $\mathbb{R} \beta$ maps trivially to $\mathrm{H}^{2}(\widetilde{\Gamma}, \mathbb{R})$. However, we have: $\psi_{\widetilde{\Gamma}}(\beta)=\pi_{\mathbb{R}}^{*}\left(\psi_{\Gamma}(\omega)\right)=\left(\pi_{\mathbb{R}}^{*} \circ\right.$ $\left.i_{\Gamma}\right)\left(\omega_{\mathbb{Z}}\right)=\left(i_{\widetilde{\Gamma}} \circ \pi_{\mathbb{Z}}^{*}\right)\left(\omega_{\mathbb{Z}}\right)$, and $\widetilde{\Gamma}$ was designed as a central extension in order to have $\pi_{\mathbb{Z}}^{*}\left(\omega_{\mathbb{Z}}\right)=0$.

REmarks. (i) The group $\widetilde{\Gamma}$ has property $(\mathrm{T})$ whenever $\Gamma$ does. Indeed, since $\psi_{\Gamma}(\omega) \neq 0$, we have $\omega_{\mathbb{Z}} \neq 0$ and the corresponding central extension does not split. The claim is now a result due to Serre [8, p. 41].

(ii) The space of pseudocharacters of $\widetilde{\Gamma}$ is isomorphic to $\operatorname{Ker}\left(\psi_{\widetilde{\Gamma}}\right)$ modulo the characters of $\widetilde{\Gamma}$; in particular, since property $(\mathrm{T})$ groups have no nonzero characters, $\widetilde{\Gamma}$ satisfies (3) if $\Gamma$ was chosen with property $(\mathrm{T})$.

(iii) The group $\widetilde{\Gamma}$ is boundedly generated whenever $\Gamma$ is so.

In conclusion, it remains to check the existence of groups $\Gamma$ satisfying (1), (2) and $\left(3^{\prime}\right)-\left(3^{\prime \prime \prime}\right)$. We obtain two families of examples from the following discussion (see also Remark (vii) below). 
Let $X$ an irreducible Hermitian symmetric space of noncompact type. Let $H:=$ $\operatorname{Isom}(X)^{\circ}$ be the identity component of its isometry group. We assume that $\pi_{1}(H)=$ $\mathbb{Z}$, that is, that $\pi_{1}(H)$ is torsion-free. We have then a central extension as in $(*)$ above, yielding a class $\omega_{H, \mathbb{Z}}$ in the 'continuous' cohomology $\mathrm{H}_{\mathrm{c}}^{2}(H, \mathbb{Z})$ (represented by a Borel cocycle); the image $\omega_{H}$ of $\omega_{H, \mathbb{Z}}$ under the natural map $\mathrm{H}_{\mathrm{c}}^{2}(H, \mathbb{Z}) \rightarrow$ $\mathrm{H}_{\mathrm{c}}^{2}(H, \mathbb{R})$ generates $\mathrm{H}_{\mathrm{c}}^{2}(H, \mathbb{R})$. For all this, see $[\mathbf{1 2}]$.

Let now $\Gamma<H$ be any lattice and let $\omega_{\mathbb{Z}}$ be the image of $\omega_{H, \mathbb{Z}}$ under the restriction map $r_{\mathbb{Z}}: \mathrm{H}_{\mathrm{c}}^{2}(H, \mathbb{Z}) \rightarrow \mathrm{H}^{2}(\Gamma, \mathbb{Z})$; thus, the corresponding central extension $\widetilde{\Gamma}$ is (isomorphic to) the preimage of $\Gamma$ in $\widetilde{H}$. Note that, so far, $\omega_{\mathbb{Z}}$ can be zero. From now on we assume that the rank of $X$ is at least two. This implies on the one hand that $H$ and $\Gamma$ have property (T) $[\mathbf{8}, 2 \mathrm{~b} .8$ and $3 \mathrm{a} .4]$; on the other hand, $\left(3^{\prime \prime}\right)$ is established in [5, Theorem 21]. Furthermore, there are isomorphisms $\mathrm{H}_{\mathrm{c}}^{2}(H, \mathbb{R}) \stackrel{\psi}{\longleftarrow}$ $\mathrm{H}_{\mathrm{cb}}^{2}(H, \mathbb{R}) \stackrel{r_{\mathbb{R}}}{\longrightarrow} \mathrm{H}_{\mathrm{b}}^{2}(\Gamma, \mathbb{R})$ (see $[\mathbf{5}]$ for the first and the vanishing theorem in $[\mathbf{1 7}]$ for the second). Thus $\left(3^{\prime}\right)$ and $\left(3^{\prime \prime \prime}\right)$ follow as well given the above discussion of the cohomology of $H$.

Finally, we investigate when $\Gamma$ (and thus $\widetilde{\Gamma}$ ) can be chosen to be boundedly generated using a result of Tavgen' $[\mathbf{2 2}$, Theorem B]. We define $\Gamma$ as integral points of a $\mathbb{Q}$-algebraic group $\underline{H}$ such that the identity component $\underline{H}(\mathbb{R})^{\circ}$ is $H=$ $\operatorname{Isom}(X)^{\circ}$. Using Tavgen's theorem requires that $\underline{H}$ be quasi-split over $\mathbb{Q}$. According to Cartan's classification [13, X.6, Table V and Section 3], the exceptional cases EIII and EVII, and the classical series DIII, are excluded because the isometry groups are not quasi-split, and a fortiori neither are their $\mathbb{Q}$-forms. Let us check that the remaining types admit quasi-split $\mathbb{Q}$-forms.

Case CI: this corresponds to Siegel's upper half-spaces $\operatorname{Sp}_{2 n}(\mathbb{R}) / \mathrm{U}(n)$. The standard symplectic forms with all coefficients equal to 1 define $\mathbb{Q}$-split algebraic subgroups of $\mathrm{SL}_{2 n}[\mathbf{3}, \mathrm{V} .23 .3]$. For each $n \geqslant 2$, the lattice $\Gamma=\operatorname{Sp}_{2 n}(\mathbb{Z}):=\operatorname{Sp}_{2 n}(\mathbb{Q}) \cap$ $\mathrm{SL}_{2 n}(\mathbb{Z})$ satisfies all the required properties. The corresponding symmetric space $X$ has rank $n$ and dimension $n(n+1)$.

Case A III: this corresponds to $\mathrm{SU}(p, q) / \mathrm{S}(\mathrm{U}(p) \times \mathrm{U}(q))$ with $p \geqslant q$. In view of the Satake-Tits diagrams [20, II Section 3], the corresponding isometry groups which are quasi-split over $\mathbb{R}$ are those for which $p=q$ or $p=q+1$. The Hermitian form $h:=\bar{x}_{1} x_{2 n}-\bar{x}_{2} x_{2 n-1}+\ldots-x_{1} \bar{x}_{2 n}$ (respectively $\bar{x}_{1} x_{2 n+1}-\bar{x}_{2} x_{2 n}+\ldots-x_{1} \bar{x}_{2 n+1}$ ), where the bar denotes the conjugation of $\mathbb{Q}(i)$, defines a $\mathbb{Q}$-form of the isometry group $\mathrm{SU}(n, n)$ (respectively $\mathrm{SU}(n+1, n)$ ). The matrices of $\mathrm{SL}_{2 n}(\mathbb{Z}[i])$ (respectively $\left.\mathrm{SL}_{2 n+1}(\mathbb{Z}[i])\right)$ preserving $h$ provide suitable groups $\Gamma$.

REMARKS. (iv) What we call bounded generation, following for example [19, Section A.2 p. 575] and [21], is what Tavgen' calls finite width, while bounded generation in $[\mathbf{2 2}]$ is defined with respect to a generating system.

(v) To have bounded generation, we restricted ourselves to arithmetic subgroups of quasi-split groups, which prevents us from constructing the groups $\Gamma$ as uniform lattices (the Godement compactness criterion requires $\mathbb{Q}$-anisotropic groups $[\mathbf{1 9}$, Theorem 4.12], which are so to speak opposite to split and quasi-split groups). The underlying deeper problem is to know whether boundedly generated uniform lattices exist [21, Introduction].

(vi) Given the cohomological vanishing results of $[\mathbf{5}, \mathbf{1 7}]$, the only possibilities for $\Gamma$ to be a lattice in (the $k$-points of) a simple group over a local field $k$ is the case we considered: $k=\mathbb{R}$, rank at least two and Hermitian structure. 
In particular, the non-Archimedean case is excluded. As far as bounded generation only is concerned, there is an even stronger obstruction in positive characteristic: any boundedly generated group that is linear in positive characteristic is virtually abelian $[\mathbf{1}]$.

(vii) A case in Cartan's classification was not alluded to above. This is the type $B D I$, corresponding to $\mathrm{SO}(p, q)^{\circ} /(\mathrm{SO}(p) \times \mathrm{SO}(q))$ with $p \geqslant q=2$. First, $\mathrm{SO}(2,2)^{\circ}$ is not simple and the associated symmetric space is not irreducible (it is the product of two hyperbolic disks). For $p \geqslant 3$, the fundamental group $\pi_{1}\left(\mathrm{SO}(p, 2)^{\circ}\right)$ has torsion since it is $\mathbb{Z} \oplus \mathbb{Z} / 2 \mathbb{Z}\left[\mathbf{1 4}\right.$, I.7.12.3], but lattices in $H=\mathrm{SO}(p, 2)^{\circ}$ still enjoy properties $(2)$ and $\left(3^{\prime}\right)-\left(3^{\prime \prime \prime}\right)$. For bounded generation, since a symmetric nondegenerate bilinear form defines a split (respectively quasi-split) orthogonal group if and only if $p-q \leqslant 1$ (respectively $p-q \leqslant 2$ ) [3, V.23.4], suitable groups $\Gamma$ are provided by lattices $\mathrm{SO}(Q) \cap \mathrm{SL}_{n}(\mathbb{Z})$, with $Q$ a nondegenerate quadratic form on $\mathbb{Q}^{n}$ of signature $(3,2)$ or $(4,2)$ over $\mathbb{Q}$.

\section{References}

1. M. Abért, A. Lubotzky and L. Pyber, 'Bounded generation and linear groups', Internat. J. Algebra Comput. 13 (2003) 401-413.

2. M. Bestrina and K. Fujiwara, 'Bounded cohomology of subgroups of mapping class groups', Geom. Topol. 6 (2002) 69-89.

3. A. Borel, Linear algebraic groups, 2nd edn, Graduate Texts in Mathematics 126 (Springer, New York, 1991).

4. M. R. Bridson and A. Haefliger, Metric spaces of non-positive curvature, Grundlehren der mathematischen Wissenschaften 319 (Springer, Berlin, 1999).

5. M. Burger and N. Monod, 'Continuous bounded cohomology and applications to rigidity theory (with an appendix by M. Burger and A. Iozzi)', Geom. Funct. Anal. 12 (2002) 219-280.

6. D. CArter and G. Keller, 'Bounded elementary generation of $\mathrm{SL}_{n}(\mathcal{O})$ ', Amer. J. Math. 105 (1983) 673-687.

7. M. Coornaert, T. Delzant and A. Papadopoulos, Géométrie et théorie des groupes: Les groupes hyperboliques de Gromov, Lecture Notes in Mathematics 1441 (Springer, Berlin, 1990).

8. P. De la Harpe and A. Valette, 'La propriété $(T)$ de Kazhdan pour les groupes localement compacts (with an appendix by M. Burger)', Astérisque 175 (1989) 158.

9. R. I. Grigorchuk, 'Some results on bounded cohomology', Combinatorial and geometric group theory (Edinburgh, 1993), London Mathematical Society Lecture Note Series 204 (Cambridge University Press, Cambridge, 1995) 111-163.

10. M. Gromov, 'Volume and bounded cohomology', Publ. Math. Inst. Hautes Études Sci. 56 (1983) 5-99.

11. M. Gromov, 'Word hyperbolic groups', Essays in group theory, Mathematical Sciences Research Institute Publications 8 (ed. S. M. Gersten; Springer, New York, 1987) 75-264.

12. A. Guichardet and D. Wigner, 'Sur la cohomologie réelle des groupes de Lie simples réels', Ann. Sci. École Norm. Sup. (4) 11 (1978) 277-292.

13. S. Helgason, Differential geometry, Lie groups, and symmetric spaces, Pure and Applied Mathematics 80 (Academic Press, New York, 1978).

14. D. Husemoller, Fibre bundles, Graduate Texts in Mathematics 20 (Springer, New York, 1966).

15. N. V. Ivanov, 'Foundations of the theory of bounded cohomology', J. Sov. Math. 37 (1987) 1090-1115.

16. J. F. Manning, 'Geometry of pseudocharacters', Geom. Topol. 9 (2005) 1147-1185.

17. N. Monod and Y. Shalom, 'Cocycle superrigidity and bounded cohomology for negatively curved spaces', J. Differential Geom. 67 (2004) 395-455.

18. L. Mosher, M. Sageev and K. Whyte, 'Quasi-actions on trees. I. Bounded valence', Ann. of Math. (2) 158 (2003) 115-164.

19. V. Platonov and A. Rapinchuk, Algebraic groups and number theory, translated from the 1991 Russian original by Rachel Rowen, Pure and Applied Mathematics 139 (Academic Press, Boston, MA, 1994). 
20. I. SATAKE, Classification theory of semi-simple algebraic groups, with an appendix by M. Sugiura, Notes prepared by Doris Schattschneider, Lecture Notes in Pure and Applied Mathematics 3 (Marcel Dekker, New York, 1971).

21. Y. Shalom, 'Bounded generation and Kazhdan's property (T)', Publ. Math. Inst. Hautes Études Sci. 90 (2001) 145-168.

22. O. I. TAVGEN', 'Bounded generability of Chevalley groups over rings of $S$-integer algebraic numbers', Izv. Akad. Nauk SSSR Ser. Mat. 54 (1990) 97-122, 221-222.

23. J. Tits, 'Systèmes générateurs de groupes de congruence', C. R. Acad. Sci. Paris Sér. A-B 283 (1976) A693-A695.

\author{
J. F. Manning \\ Mathematics Department \\ MC253-37 \\ California Institute of Technology \\ Pasadena \\ CA 91125 \\ USA
}

manning@caltech.edu

\author{
N. Monod \\ Department of Mathematics \\ University of Chicago \\ 5734 South University Avenue \\ Chicago \\ IL 60637 \\ USA \\ monod@math.uchicago.edu
}

\author{
B. Rémy \\ Institut Camille Jordan \\ UFR de Mathématiques \\ UMR 5208 CNRS/Lyon 1 \\ Bâtiment Jean Braconnier \\ 21 Rue Claude Bernard \\ Université Claude Bernard Lyon 1 \\ 69622 Villeurbanne cedex \\ France \\ bertrand.remy@ujf-grenoble.fr
}

